\title{
RDUS
}

Revue de DROIT

UNIVERSITÉ DE SHERBROOKE

Titre : $\quad$ CONSTRUCTION INTERNATIONALE ET RESPONSABILITÉ CIVILE DU CONSTRUCTEUR : LES PERSPECTIVES EN DROIT INTERNATIONAL PRIVÉ QUÉBÉCOIS

Auteur(s) : $\quad$ Gérald GOLDSTEIN

Revue : $\quad$ RDUS, 1991-1992, volume 22, numéro 2

Pages: $\quad 291-342$

ISSN : $\quad 0317-9656$

Éditeur : $\quad$ Université de Sherbrooke. Faculté de droit.

URI : $\quad$ http://hdl.handle.net/11143/13427

DOI : $\quad$ https://doi.org/10.17118/11143/13427 
Page vide laissée intentionnellement. 


\section{CONSTRUCTION INTERNATIONALE ET RESPONSABILITÉ CIVILE DU CONSTRUCTEUR: LES PERSPECTIVES EN DROIT INTERNATIONAL PRIVÉ QUÉBÉCOIS}

par Gérald GOLDSTEIN

Cette étude aborde la question de la loi applicable au contrat international de construction, jusqu'à présent négligée en droit international privé québécois, en premier lieu, essentiellement du point de vue de la responsabilité du constructeur pour vice du sol ou de construction vis-à-vis du maître d'ouvrage et, accessoirement, du point de vue des rapports entre ces deux parties, les sous-traitants et les assureurs. En second lieu, il y est fait état de la condition des sociétés étrangères de construction au Québec.

Si les questions de la loi applicable au fond des contrats de construction internationale et à la responsabilité contractuelle et extracontractuelle de droit commun qui en découlent ne posent pas de problème particulier, la difficulté centrale touche la qualification de la responsabilité particulière découlant en droit québécois de l'article 1688. En effet, cette qualification, contractuelle, légale, ou extracontractuelle, n'est pas certaine en droit interne. Or, elle a pour conséquence directe le choix de la règle de conflit en droit international privé et donc celui de la loi applicable.

La qualification de la question permettrait aux parties d'écarter l'application de l'article 1688 C.c., même si la construction avait lieu au Québec, ce qui irait à l'encontre de la politique préventive de protection de la population québécoise que cette disposition veut promouvoir. Cependant, comme on la considère généralement d'ordre public en droit interne, on pourrait quand même écarter une solution fondée sur une loi étrangère en invoquant sa contrariété à la conception fondamentale que l'article 1688 C.c. exprime, en faisant appel, au cas par cas, à l'exception d'ordre public, telle

*. Professeur à la Faculté de droit de l'Université de Montréal. L'auteur tient à remercier Mme Anne Malfait (étudiante au Barreau) de son assistance efficace dans la recherche relative à la partie IV de cette étude, ainsi que Me Claire Moffet (avocate et chargée de cours) pour les informations qu'elle a eu la gentillesse de lui fournir sur cette même partie. 
qu'on l'entend dans l'ordre international. Plus radicalement, on pourrait aussi considérer cette disposition comme une loi de police de façon à l'appliquer $\grave{a}$ tous les contrats donnant lieu à des constructions au Québec, sans jamais tenir compte de la loi choisie par les parties pour régir leurs rapports.

La qualification légale ou extracontractuelle de cette question permettrait d'éviter ces procédés exceptionnels, tout en faisant respecter la politique interne, puisque les parties n'auraient plus la possibilité de choisir la loi gouvernant cette responsabilité. Pour ces motifs, l'auteur opte pour cette solution, en constatant que la jurisprudence de droit interne se prononce en ce sens, que la Cour d'appel, dans un litige de droit international privé, a adopté une solution qui pourrait s'expliquer de cette façon et, enfin, que l'article 35 de la Loi sur la qualification des entrepreneurs de construction exprime une approche législative territorialiste de la question. Mais le problème consiste alors à déterminer le facteur de rattachement approprié. L'auteur propose une règle de conflit à caractère substantiel comportant un facteur de rattachement alternatif, se traduisant par un choix entre la loi du lieu de la construction et celle de l'établissement principal du constructeur, selon celle qui serait la plus favorable au maître d'ouvrage, ce qui aurait aussi l'avantage de protéger le public.

Dans les deux hypothèses de qualification, il faut déterminer le domaine d'application de la loi régissant la responsabilité du constructeur

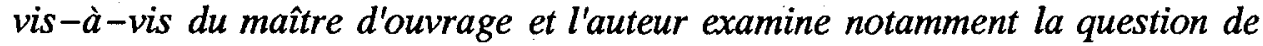
la loi relative au délai ou à la prescription de l'action en regard des règles matérielles complexes contenues aux articles 2190 et 2191 C.c.

Par ailleurs, il s'efforce de préciser la loi applicable aux rapports de sous-traitance, notamment entre le sous-traitant et le maître d'ouvrage. Il examine aussi les nombreuses règles de police régissant le contrat d'assurance dans la même optique du contrat de construction internationale.

Enfin, l'étude s'attache à présenter une vue d'ensemble des règles régissant l'activité des sociétés étrangères de construction au Québec, notamment au plan des procédures de soumission et d'adjudication, pour les contrats de construction privés comme pour ceux donnés par les organismes publics. 
This study discusses issues pertaining to the law that applies to international construction contracts, an area that up to now has received little attention in Quebec private international law. The first part deals with the builder's responsibility towards the client for defects of construction or of the land. It also examines, the relationship between the client and the builder, the subcontractors and the insurers. The second part describes the conditions that apply to foreign construction companies in Quebec.

Even though the legal questions that pertain to international construction contracts and to the contractual and extracontractual responsibilities under common law pose no unusual problems, the primary difficulty concerns the liability which results from article 1688 of the Civil Code. In fact, the determination whether this responsibility is contractual, legal or extracontractual remains uncertain in internal law. As a result, one must choose the rule in private international law that would govern this conflict and then determine which law in fact applies.

To hold that this responsibility is contractual would exclude the application of article 1688 C.C. even if the construction took place in Quebec. This would be contrary to the preventive aim of this article, which is intended to protect the public. However, since the article in question is generally considered to be of public order in internal law, one could eliminate the application of a foreign law by invoking that the latter contradicts the basic concepts expressed in article 1688 C.C. and by using the public order exception clause. As a more radical approach it could be argued that this article constitutes a law of police and should apply to all contracts that pertain to construction in Quebec, thereby excluding any other law chosen by the parties to govern their relationships.

The qualification that liability is legal or extracontractual in such matters permits one to avoid recourse to these exceptional measures, while at the same time ensuring respect for internal policies, because the parties would no longer have the possibility of choosing the law that would govern liability. For these reasons, the writer, noting that this is the point of view held by jurisprudence, recommends this solution. The Court of Appeal, in a case involving private international law, decided the issue in a manner consonant with this approach and section 35 of an Act respecting the qualifications of construction contractors expresses a similar territorialist legislative approach to the question. Thus the problem then becomes one of merely finding the 
appropriate connecting factor. The writer proposes a substantive conflict rule that includes alternative criteria for eligibility, based on either the location of the construction project or else on the situation of the principal place of business of the builder, according to whichever one would be the most favorable for the client. This would also have the advantage of offering the greatest protection to the public.

In effecting a characterization according to these alternative connecting factors, one must determine the pertinent law governing the responsibility of the contractor toward the owner. For instance, the writer examines the prescriptive delays applicable to law-suits in light of the material rules set out in articles 2190 and 2191 C.C.

In addition, he seeks to determine the law pertaining to subcontracts and in particular, to the relationship between subcontractors and the client. He also examines the numerous laws of police that govern insurance contracts in the same field of international construction contracts.

Finally, this study aims at presenting an overview of the rules that regulate the activities of foreign construction companies operating in Quebec, with emphasis on procedures for the submission of tenders and the awarding of contracts by private parties as well as by public bodies. 


\section{SOMMAIRE}

I. La détermination de la loi applicable aux contrats internationaux de construction et à la responsabilité civile qui en découle

A. La loi applicable au fond et à la forme des contrats de

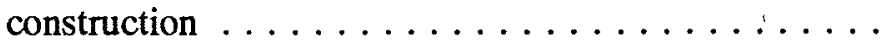

B. La loi applicable à la responsabilité civile du constructeur . . . . . . . . . . . . . . . .

1. Responsabilité civile contractuelle et quasi-délictuelle de droit commun . . . . . . . . . . . 303

2. Responsabilité spéciale pour vice de construction .................. 305

a. La thèse de la qualification contractuelle ............... 306

b. La thèse de la qualification extracontractuelle ............. 306

i. Application de la double règle quasi délictuelle: problème de la détermination du lieu du délit

ii. Choix d'un rattachement spécifique $\ldots \ldots \ldots \ldots \ldots 311$

c. La qualification en droit positif in-

C. Le domaine de la loi applicable à la responsabilité civile du constructeur

II. L'ordre public international et la responsabilité civile du constructeur pour vice de construction

A. La responsabilité civile du constructeur pour vice de construction est-elle d'ordre public international? . . . 324

B. Le domaine des articles 1688 et 2259 C.c. s'ils étaient considérés d'ordre public international . . . . . . . 327 
III. Quelques autres aspects conflictuels du droit international privé de la construction internationale . . . . . . . . . 329

A. La loi applicable à la sous-traitance $\ldots \ldots \ldots \ldots . \ldots 330$

B. La loi applicable au contrat d'assurance ........ 333

IV. La condition des sociétés de construction étrangères au Québec 


\section{Introduction}

Malgré l'importance du contrat international de construction, les éléments permettant d'en déterminer le régime général en droit international privé québécois sont quasiment inexistants. La jurisprudence n'a pas eu l'occasion d'en traiter directement, probablement en raison du recours à l'arbitrage généralement prévu dans ce genre de contrat. La législation ne prévoit que quelques dispositions éparses relativement aux sociétés «étrangères», c'est-à-dire non «Tésidentes» au Québec. Elles visent à assurer le respect du droit québécois en exigeant une licence à défaut de quoi elles sont exclues des marchés de travaux publics. La doctrine québécoise ignore en général le domaine ${ }^{1}$.

Les sociétés étrangères agissent essentiellement au Québec en y créant des filiales québécoises ou en participant à des joint ventures, ce qui leur permet d'agir sous le couvert de sociétés québécoises. Néanmoins, de temps en temps un certain nombre de travaux sont confiés à des entreprises canadiennes d'autres provinces, à des entreprises américaines ou européennes, obligées d'obtenir une licence québécoise pour construire au Québec. Elles doivent alors établir un domicile québécois, c'est-à-dire posséder au moins une adresse ou un établissement susceptible d'y recevoir des significations, des mises en demeure, etc. Par ailleurs, certaines compagnies québécoises se sont déjà franchement lancées dans le marché des contrats de construction à l'étranger, notamment dans le cadre d'accords de coopération avec des pays en voie de développement. Les contrats prévoient en général une clause d'arbitrage, mais la question de la loi applicable n'y est pas nécessairement

1. Voir cependant: M, DESCHAMPS, «L'autonomie de la volonté dans la détermination de la loi contractuelle et du tribunal compétent», (1990) 24 R.J.T. 471, p. 474-475. Voir aussi, sur le contenu et la réglementation des contrats internationaux de construction et d'entreprise, en général: V. KARIM, Les contrats de réalisation d'ensembles industriels et de transfert de technologie, Cowansville, Yvon Blais, 1987; A. COSSETTE, «Certaines difficultés de la phase précontractuelle dans les contrats internationaux "clés en main"», (1988) $90 R$ du N. 384; M. HANNANI, «Les garanties contractuelles dans les transactions internationales comportant la foumiture de biens ou de services", (1985) 26 C. de D. 633, p. 646 et suiv. 
prévue, bien que les pays en voie de développement essayent parfois d'imposer leur loi locale.

À partir de ce cadre très ténu, nous nous efforcerons d'apporter des éléments de réponse aux principales questions soulevées par la matière. Nous procéderons en examinant d'abord la loi applicable entre maître d'ouvrage et constructeur, aux contrats de construction et à la responsabilité civile qui en découle par le biais de la méthode conflictuelle classique (I); puis nous étudierons les dispositions québécoises qui pourraient intervenir impérativement en ce domaine en tant que loi de police ou au nom de l'ordre public (II), ainsi que les règles matérielles régissant la condition des sociétés étrangères de construction au Québec (IV). Nous aborderons aussi quelques autres questions accessoires au contrat de construction (III): celles de la loi applicable au contrat de sous-traitance et aux assurances en ce domaine.

\section{La détermination de la loi applicable aux contrats internationaux de construction et à la responsabilité civile qui en découle}

Le contrat de construction ne présente pas d'originalité au point de vue de la compétence internationale des tribunaux québécois: les règles communes s'appliquent $^{2}$.

2. Voir à ce sujet: E. GROFFIER, Précis de droit international privé québécois, 4e éd., Cowansville, Yvon Blais, 1990, (ci-après Précis) no 254 et suiv., p. 251; J.-G. CASTEL, Droit international privé québécois, Toronto, Butterworths, 1980, p. 671 et suiv.; J. TAIPIS, «Notions élémentaires de droit international privé québécois», (1977) CP. du N. 115, no 7 et suiv., p. 128. En droit québécois, le tribunal est compétent pour entendre une action personnelle à caractère patrimonial en vertu de l'article 68 du Code de procédure civile. Celui-ci retient comme chefs de compétence alternatifs: 1) le domicile réel ou élu du défendeur au Québec, 2) la résidence du défendeur ou la possession de biens au Québec, 3) le fait que «toute la cause d'action» $y$ ait pris naissance et enfin, 4) la conclusion du contrat au Québec.

Ainsi, il suffira que le maître d'ouvrage ou l'entrepreneur soient domiciliés, résidents ou possèdent des biens (comme des machines, etc.) au Québec, s'ils sont défendeurs, pour entraîner la compétence du juge québécoijs. Par «biens», on entend aussi les droits personnels, comme les créances (GROFFIER, Précis, no 258, p. 253). Jusqu'à présent, la jurisprudence n'exige pas que les biens suffisent à couvrir le montant de la demande (Voir en ce sens: CASTEL, id., p. 680; comparez, avec Southern Pacific Co. c. Botner, [1973] R.P. 97 (C.A.); Can. Unicofam Ltd c. Griegce \& Autres, [1974] C.S. 402). Le chef de 


\section{A. La loi applicable au fond et à la forme des contrats de construc- tion $^{3}$}

En droit québécois, les conditions de formation et les effets des contrats sont soumis à la règle de conflit contenue dans l'article 8 C.c. ${ }^{4}$ Les contrats de construction ne font pas exception à cette règle qui retient en principe la loi d'autonomie. Le juge québécois applique la loi expressément choisie par les parties, à défaut, il recherche des indices de leur intention implicite dans le contrat et dans les circonstances extemes qui l'entourent ${ }^{5}$. À défaut de pouvoir découvrir cette intention, il est présumé que les parties

compétence fondé sur l'existence de «toute la cause d'action» au Québec a été assoupli par la jurisprudence. Dans l'affaire Wabasso (Wabasso Ltd c. National Drying Machinery Co., [1981] 1 R.C.S. 578, inf. [1979] C.A. 279. Voir: H.P. GLENN, «Toute la cause d'action et de la compétence internationale», (1982) 27 McGill L.J. 793; E. GROFFIER, "Chroniques régulières. Toute la cause d'action", (1981) $41 R$. du B. 1102; GROFFIER, Précis, no 261, p. 254), la Cour suprême du Canada a décidé qu'il suffisait qu'y soient localisés tous les faits dont la preuve est essentielle pour établir la prétention, telle que libellée. La présence de certains éléments à l'étranger n'empêchera donc pas le juge québécois de se déclarer compétent. Par ailleurs, il en sera de même si le contrat est conclu au Québec. Indépendamment des cas prévus à l'article 68 C.p.c., les parties peuvent s'entendre pour donner compétence aux tribunaux québécois (Alimport c. Victoria Transport Ltd, [1977] 2 R.C.S. 858; GROFFIER, Précis, no 268, p. 261). Enfin, les tribunaux québécois refusent de se déclarer incompétents en faveur d'un autre tribunal si l'un des chefs de compétence de l'article 68 se trouve satisfait (Franjan Productions Inc. c. Security Investment Trust Co., [1975] C.S. 1140; Goodwin's Ltd c. Furness Withy Co. Ltd, (1916) 17 R.P. 118 (C.S.); GROFFIER, Précis, no 268, p. 261), bien qu'ils acceptent, curieusement, de se désister en faveur d'un tribunal arbitral (Zodiac International Productions Inc. c. The Polish People's Republic, [1983] R.D.J. 277 (C.S.C.); GROFFIER, Précis, no 269, p. 261).

3. Sur ce sujet, voir généralement: J.P. REMERY, «Remarques sur le conflit des lois applicables au contrat international de construction d'immeuble», D.1985 chron.43, p. 257; R. CHEMALY, «Louage d'ouvrage et d'industrie», dans Rep. droit international, Dalloz, 1968 (et mises à jour).

4. Art. 8 C.c.: «Les actes s'interprètent suivant la loi du lieu où ils sont passés, à moins qu'il n'y ait quelque loi à ce contraire, que les parties ne s'en soient exprimées autrement, ou que, de la nature de l'acte, ou des autres circonstances, il n'apparaisse que l'intention a été de s'en rapporter à la loi d'un autre lieu; auxquels cas il est donné effet à cette loi, ou à cette intention exprimée ou présumée.»; voir GROFFIER, Précis, op. cit., supra, note 2, no 185 et suiv., p. 185.

5. Voir Smith Transport Ltd c. IN-TRA-CO. Inc., [1974] C.S. 265. 
ont voulu se référer à la loi du lieu de conclusion du contrat. Cette règle de conflit s'applique évidemment sous la réserve, prévue dans l'article 8 , de l'intervention des lois de police.

Par conséquent, en l'absence de choix exprès, il n'est pas certain que le juge québécois applique la loi du lieu d'exécution du contrat international d'entreprise, si celui-ci est conclu dans un autre lieu.

Le projet de Code civil de $1991^{6}$ modifie considérablement cette règle puisqu'il s'inspire des solutions de la Convention de Rome de juin 1980 sur la loi applicable aux obligations contractuelles ${ }^{7}$. En l'absence de choix exprès, le futur droit québécois reconnaîtra donc le principe de la loi du lieu avec lequel le contrat présente «les liens les plus étroits». L'article 3113 du Projet prévoit que les «liens les plus étroits» seront présumés exister avec l'État du lieu de la résidence habituelle ou de l'établissement, s'il s'agit d'une entreprise, de la partie qui doit fournir la prestation caractéristique. Il s'agira donc de la loi du lieu d'établissement de l'entrepreneur, ou du sous-traitant, pour le contrat de construction, ce qui ne nous semble pas la loi la plus pertinente.

Cependant, tout comme la Convention de Rome, le futur droit québécois comprendra une clause échappatoire (art. 3082 du Projet) qui, exceptionnellement, permettra de déroger à cette présomption pour désigner probablement la loi du lieu d'exécution des travaux ${ }^{8}$, si celle-ci a un lien

6. Projet de Loi 125, Code civil du Québec, L.Q. 1991, c. 64, Assemblée nationale, 34 Législature, première session, Éditeur officiel du Québec, 1991.

7. Voir son texte à J.O., C.E. no L.266 du 9 oct. 1980; (1980) 69 Rev. crit. dr. internat. privé 875; (1981) J.D.I. 218; (1981) 17 Rev. Trim. D. Europ. 394.

8. Sur la clause échappatoire, voir C. DUBLER, Les clauses d'exception en droit international privé, Georg, Genèves, 1983. Voir aussi: J.A. TALPIS et G. GOLDSTEIN, «Analyse critique de l'Avant-projet de loi du Québec en droit international privé», (1989) $91 R$. du N. 293, p. 327-331. Cette exception pourrait bien se transformer en une future nouvelle règle de conflit propre aux contrats de construction, par la force des précédents. Mais rien ne le garantit, d'autant plus qu'essentiellement la clause échappatoire n'est censée intervenir que ponctuellement, sans nécessairement imposer qu'on se réfère aux décisions antérieures en tant qu'autorités.

On doit toutefois faire remarquer, qu'étant donné le petit nombre [total] de décisions rendues au Québec en droit international privé, il est probable que l'intervention de la 
beaucoup plus étroit avec le contrat que celle du lieu de résidence de l'entrepreur.

Quant au domaine de la loi applicable, il comprend certainement l'interprétation et la responsabilité contractuelle ${ }^{9}$ résultant du contrat de construction, mais non la responsabilité extra-contractuelle, régie par une autre règle de conflit que nous exposerons plus bas, ni la capacité de contracter $^{10}$ qui résulte de l'application de la loi du lieu de l'établissement de l'entreprise (équivalent du domicile) selon l'article 6 al. 4 C.c.

En pratique, les compagnies québécoises engagées dans la construction à l'étranger composent le contenu de leur contrat en combinant des «clausesmaison» avec des clauses-types de la FIDIC $^{11}$ et du Guide juridique de la C.N.U.D.C.I. pour l'établissement de contrats internationaux de construction d'installations industrielles ${ }^{12}$.

Cependant, plus généralement, il n'y a pas encore au Canada d'acceptation de la lex mercatoria, applicable au contrat sans aucune référence, ni même de reconnaissance expresse de la notion en tant que telle. Toutefois, en droit maritime, la Cour suprême du Canada a invoqué la nécessité d'interpréter les principes en tenant compte du droit international ${ }^{13}$, pour écarter le droit civil au profit de la common law ${ }^{14}$, au motif que:

clause échappatoire, pour tous les cas de contrat de construction, y soit considérée comme «exceptionnelle».

9. GROFFIER, Précis, op. cit., supra, note 2, no 190, p. 190.

10. Ibid.

11. Par exemple: FIDIC, Guide to the Use of FIDIC Conditions of Contract for Electrical and Mechanical Works, 3rd ed., FIDIC, Lausanne, 1988; FIDIC, Guide to the Use of FIDIC Conditions of Contract for Works of Civil Engineering Construction, 4th ed., FIDIC, Lausanne, 1989.

12. C.N.U.D.C.I., Publication des Nations-Unies, New York, 1988.

13. Q.N.S. Paper Co. c. Chartwell Shipping Ltd, [1989] 2 R.C.S. 683, p. 691-693 par le juge McLachlin. Voir aussi TTO-International Terminal Operators Ltd C. Miida Electronics Inc., [1986] 1 R.C.S. 752.

14. Voir à ce sujet les critiques de A. BRAËN, «L'arrêt ITO-International Operators Ltd c. Miida Electronics Inc., ou comment écarter l'application du droit civil dans un litige maritime au Québec», (1987) 32 McGill L.J. 386; H.P. GLENN, «Maritime Law Federal Court Jurisdiction - Canadian Maritime Law - Relationship to Civil and 
«Dans les affaires maritimes ou d'amirauté, nous traitons de commerce international. Il existe de bonnes raisons de promouvoir l'uniformité dans ce domaine, ainsi que le plus haut degré possible de certitude. ${ }^{15}$ " mentaire ${ }^{16}$.

La Cour suprême a invoqué le même principe en matière de crédit docu-

De plus, le nouvel article 940.6 du Code de procédure civile québécois prévoit que lorsqu'un arbitrage met en cause des intérêts du commerce extraprovincial ou international, la convention d'arbitrage doit s'interpréter, à titre supplétif, en tenant compte de la Loi type sur l'arbitrage commercial international, adoptée le 21 juin 1985 par la C.N.U.D.C.I. ${ }^{17}$

Par ailleurs, on ne discerne pas non plus au Canada d'application consciente de la «théorie du tronc commun»" même si la Cour suprême, par exemple, en raison des particularités du système juridique canadien, utilise parfois l'argument selon lequel le droit civil et la common law ont un contenu commun sur un sujet particulier, d'un point de vue comparatiste ${ }^{19}$.

Il faut mentionner enfin que la validité formelle du contrat dépend, par application de l'article 7 C.c., de la loi du lieu de passation de l'acte. Mais cette règle de conflit est «supplétive», car elle admet alternativement d'autres

Common Law: ITO-International Terminal Operators Ltd v. Miida Electronics Inc.», (1987) 66 Rev. Bar. Can. 360; G. LEFEBVRE et N. TAMARO, «La Cour suprême et le droit maritime: la mise à l'écart du droit civil québécois est-elle justifiable?» (1991) 70 Rev. Bar. Can 121.

15. ITO-Intermational Terminal Operators Ltd, précité, supra, note 13, p. 788.

16. Banque de Nouvelle-Écosse c. Angelica-Whitewear. Ltd, [1987] 1 R.C.S. 59, p. 83.

17. À ce sujet, voir notamment: A PRUJINER, «Les nouvelles règles de l'arbitrage au Québec», (1987) Rev. Arb. 425; J.E.C. BRIERIEY, «Une loi nouvelle pour le Québec en matière d'arbitrage», (1987) $47 R$. du B. 259; S. THUILLEAUX, L'arbitrage commercial au Québec: droit interne, droit international privé, Montréal, Yvon Blais, 1991.

18. M. RUBINO-SAMMARTINO, «Le "tronc commun" des lois nationales en présence (Réflexion sur le droit applicable par l'arbitre international)», (1987) Rev. Arb. 133.

19. Voir ainsi: Q.N.S. Paper Co. c. Chartwell Shipping Ltd, précité, supra, note 13. 
facteurs de rattachement, comme, probablement, le lieu d'exécution du contrat, ou, plus généralement, le rattachement par la loi régissant le fond de l'acte ${ }^{20}$.

\section{B. La loi applicable à la responsabilité civile du constructeur}

Il s'agit de la question la plus intéressante, du point de vue du droit international privé québécois, et peut-être aussi de l'une des plus délicates, dans le domaine de la construction internationale. Le problème central est celui de la qualification, contractuelle ou extracontractuelle, des différents types de responsabilité encourue à la suite d'un contrat de construction, car le contenu même des diverses règles de conflit est connu.

\section{Responsabilité civile contractuelle et quasi-délictuelle de droit com- mun}

La responsabilité contractuelle, (ce qui inclut la validité des clauses d'exclusion de cette responsabilité, selon la jurisprudence) ${ }^{21}$ dépend de la loi contractuelle. Quant à la responsabilité civile délictuelle et quasi-délictuelle québécoise, elle est soumise par la jurisprudence à la double règle de conflit tirée de la common law anglaise, notamment de l'arrêt Phillips c. Eyre ${ }^{22}$. Elle prévoit que le demandeur au Québec en responsabilité obtient gain de cause conformément à la loi québécoise (intervenant en tant que loi du for) si 1) l'acte préjudiciable justifie une action en dommages et intérêts selon la loi québécoise et 2) s'il constitue un acte injustifiable, susceptible de sanction civile ou même simplement pénale ${ }^{23}$ selon la loi du lieu où il a été commis $(\text { lex loci delicti })^{24}$.

20. Toutefois la question ne présente pas d'intérêt particulier car les litiges en matière de construction ne concernent que le fond du contrat et la responsabilité.

21. Voir la jurisprudence citée par le professeur GROFFIER, (Précis, op. cit., supra, note 2, no 199 , p. 196 , note 31 ).

22. (1870) L.R. 6 Q.B. 1.

23. Machado c. Fontes, [1897] 2 Q.B. 231, repris au Canada dans McLean c. Pettigrew, [1945] R.C.S. 62; voir cependant Lieff c. Palmer, (1937) 63 Q.B. 278.

24. O'Connor c. Wray, [1930] R.C.S. 231. Voir GROFFIER, Précis, op. cit., supra, note 2, no 224 et suiv., CASTEL, op. cit., supra, note 2, p. 447 et suiv.

Pourtant, l'article 6 al. 3 du Code civil québécois prévoit que: «Les lois du Bas Canada relatives aux personnes sont applicables à tous ceux qui s'y trouvent, même à ceux qui 
Dans le contrat de construction, la responsabilité civile quasi délictuelle du constructeur envers le maître d'ouvrage, les fournisseurs, les sous-traitants, les ouvriers et les tiers absolus suivra donc cette double règle.

n'y sont pas domiciliés; [...]»

Cette disposition, inspirée de l'article 3 al. 1 du Code civil français, était destinée à exprimer l'adoption en droit international privé québécois du principe général du territorialisme (voir à ce sujet la pénétrante analyse du professeur P.-A. CRÉPEAU, «De la responsabilité civile extra-contractuelle en droit international privé québécois», (1961) 39 Can. Bar. Rev. 3), auxquelles certaines dérogations particulières étaient prévues, notamment la soumission du statut personnel à la loi du domicile (art. 6 al. 4 C.c.).

La jurisprudence a d'abord reconnu ce point de vue (voir la jurisprudence citée par P.-A. CRÉPEAU, (ibid) et par J.-G. CASTEL, op. cit., supra, note 2, p. 449, note 7) en matière de responsabilité quasi-délictuelle, en bilatéralisant la lex loci delicti. Mais la Cour suprême du Canada a modifié cette règle en 1930 ( $O^{\prime}$ Connor c. Wray, ibid) au motif, discutable, que les règles de conflit québécoise et anglaise en la matière étaient identiques et que la règle québécoise ne devait pas être «plus généreuse» que celle résultant de la common law anglaise.

Malgré les critiques vigoureuses de la doctrine québécoise (voir notamment P.-A. CRÉPEAU, ibid), la jurisprudence applique maintenant la règle de common law (McLean c. Pettigrew, précité, supra, note 23; Samson c. Holden, [1963] R.C.S. 373; Gauthier c. Bergeron, [1973] C.A. 77, p. 79), à l'exception de quelques décisions (Lieff c. Palmer, précité, supra, note 23; Bussières c. Pelissier, C.S. Québec, 84577, 23 mai 1958, (1970) 11 C. de D. 373, (1967) 13 McGill.LJ. 331).

Cependant, la règle de conflit anglaise exige maintenant, en principe, que l'acte soit susceptible d'entraîner une action civile selon les deux lois - lex fori et lex loci delicti (règle dite de la double «actionabilité») mais elle a aussi évolué vers la proper law of the tort (Chaplin c. Boys, [1971] A.C. 356 (H.L.), Sayers v. International Drilling Co. N.V., [1971] 1 W.L.R. 1176, 1180; Church of Scientology of America v. Metropolitan Police Commissioner, (1976) 120 Sol. Jo. 690; Coupland v. Arabian Gulf Petroleum Co., [1983] 3 All E.R. 226; Dicey and Morris on the Conflicts of Laws, 11 ${ }^{\circ}$ éd., par L. COLLINS, (éd.), vol. 2, London, Stevens \& Sons, 1987, Rule 205, p. 1365 et s.; CHESHIRE and NORTH, Private International Law, $11^{\circ}$ ed., par P.M. NORTH et J.J. FAWCETT, London, Butterworths, 1987, p. 535-536), c'est-à-dire vers une approche flexible prenant en considération l'ensemble des rattachements pour tenir compte, dans chaque cas, des plus significatifs pour l'espèce (voir J.H.C. MORRIS, «The Proper Law of a Tort», (1951) 64 Harv. L. Rev. 881).

Récemment, la Cour d'appel de l'Ontario a adopté la même méthode Grimes c. Cloutier, (1989) 61 D.L.R. (4th) 505, p. 524-525; voir surtout Préfontaine Estate c. Frizzel, (1990) 65 D.L.R. (4 th) 275, p. 281; voir G. GOLDSTEIN, «Droit international privé (chronique)», (1988) 5 R.Q.D.I. 416, p. 421-422. Il reste à voir l'incidence que l'adoption de cette nouvelle règle de conflit en Ontario aura au Canada, et au Québec en particulier. 
Dans le futur code civil, elle sera remplacée par le principe de la compétence d'une loi unique, celle du lieu du délit ${ }^{25}$. Ce lieu sera celui de la survenance du fait générateur du préjudice, s'il n'est pas dissocié de celui du dommage: on donnerait alors préférence au dernier (sauf si l'auteur ne devait pas prévoir que le préjudice allait y apparaître). Toutefois si les deux parties étaient résidentes du même pays ou si elles y étaient domiciliées, on appliquerait cette loi commune. En principe, en matière de responsabilité quasi délictuelle du constructeur, la loi québécoise ou étrangère du lieu de construction devrait donc s'appliquer si le dommage y est subi (sauf si le constructeur et la victime sont résidents ou domiciliés ailleurs).

Mais qu'en est-il de la responsabilité spéciale ou «garantie» décennale (comme en certains pays) ou quinquennale (comme au Québec dans l'article 1688 C.c.) du constructeur pour les vices de construction et les vices du sol, qui se poursuit après la réception des travaux, indépendamment de la relation contractuelle?

\section{Responsabilité spéciale pour vice de construction}

La question présente notamment un intérêt lorsque les législations en présence comprennent des conditions et des durées de garantie différentes, des domaines d'application différents (application aux architectes, aux ingénieurs?) ou encore ne concordent pas quant à la transmissibilité de l'action en garantie ou quant à la légalité des clauses d'exclusion de responsabilité.

En général, la jurisprudence québécoise qualifie les prétentions de droit selon la loi du for ${ }^{26}$. Par conséquent, il faut déterminer comment le droit civil interne québécois qualifie cette responsabilité pour vice de construction pour savoir si le juge appliquera sa règle de conflit contractuelle (loi d'autonomie) ou extracontractuelle.

25. Projet de loi 125, précité, supra, note 6, article 3126.

26. Gauthier c. Bergeron, précité, supra, note 24; voir cependant Samson c. Holden, précité, supra, note 24 (affirmation selon laquelle la qualité pour ester en justice constitue une question de procédure); Pouliot c. Cloutier, [1944] R.C.S. 284. 


\section{a. La thèse de la qualification contractuelle}

Si l'on opte pour la qualification contractuelle, les parties devraient pouvoir échapper à cette responsabilité, puisqu'en vertu de l'article 8 du Code civil elles peuvent choisir la loi applicable au contrat, si cette loi ne la connaît pas ou reconnaît la validité des clauses de non-responsabilitée ${ }^{27}$.

Mais il est possible que, même dans cette hypothèse de qualification contractuelle (comme dans toute autre hypothèse de qualification, d'ailleurs), la solution québécoise soit considérée si impérative qu'elle donne lieu à l'intervention de ses dispositions (article 1688 et 2259 C.c.) soit directement à titre de loi de police sans passer par l'étape de détermination de la loi applicable selon l'article 8 C.c., du moins quant au principe de cette responsabilité, soit indirectement, après que le juge ait écarté la loi étrangère applicable en raison de sa contrariété avec les conceptions fondamentales du Québec, en ionvoquant l'exception d'ordre public ${ }^{28}$. Nous traiterons plus loin de cette possibilité.

\section{b. La thèse de la qualification extracontractuelle}

$\mathrm{Si}$, au contraire, on opte pour la qualification extracontractuelle, il faut alors encore déterminer le facteur de rattachement de la règle de conflit. Plusieurs solutions sont possibles. On peut soit appliquer la règle de conflit jurisprudentielle applicable aux quasi-délits (i), ce qui pose le problème du choix du lieu du délit, ou chercher un rattachement spécifique (ii), en se fondant sur le principe général de territorialité, en invoquant le caractère extracontractuel ou quasi contractuel de la responsabilité.

27. Voir en ce sens: M. DESCHAMPS, loc. cit., supra, note 1, p. 479.

28. Sur la notion de loi de police ou d'application immédiate, voir: CASTEL, op. cit., supra, note 2, p. 94; GROFFIER, Précis, op, cit., supra, note 2, no 28-32, p. 32-37; J.A. TALPIS, «Legal Rules Which Determine their Own Sphere of Application: A Proposal for their Recognition in Quebec Private International Law», (1982-83) 17 R.J.T. 201. Pour la différence entre loi de police et l'exception d'ordre public, voir aussi: G. GOLDSTEIN, «L'ordre public en droit international privé de la protection du consommateur: Commentaire de G.M.A.C. c. Arnold», (1984) 30 McGill L.J. 143, p. 153-157. 


\section{i. Application de la double règle quasi délictuelle: problème de la détermination du lieu du délit}

Une première solution consiste à appliquer simplement la double règle propre à la responsabilité quasi-délictuelle de droit commun. Si la construction est érigée au Québec, la loi québécoise s'appliquera à la fois à titre de loi du for et en tant que loi du lieu du «délit». Mais, question générale et complexe, faut-il tenir compte de la loi du lieu de l'acte fautif ou de celle du lieu où le préjudice est subi $^{29}$ ? La question pourrait se présenter, par exemple, lors d'une action intentée au Québec pour vice de construction d'un édifice québécois dont certaines parties auraient été préfabriquées et montées au lieu, étranger, du siège principal de la société constructrice. On peut aussi songer au cas d'une construction érigée par un constructeur québécois à l'étranger.

La Cour suprême du Canada a décidé, notamment en matière de responsabilité du fabricant pour vice de fabrication, dans des affaires non-québécoises cependant ${ }^{30}$, qu'il fallait retenir comme facteur de rattachement le lieu où se produit l'événement essentiel, c'est-à-dire celui où le préjudice est subi ${ }^{31}$. En serait-il de même pour le droit québécois? “'̀ notre avis, tout laisse à penser que oui.

En ce sens, on peut d'abord citer le jugement rendu dans l'affaire $A$. Côté et Frères Ltée c. Laboratoires Sagi Inc. ${ }^{32}$

Le professeur Popovici fait cependant remarquer que le lieu du fait générateur présente au moins autant, sinon plus d'arguments en sa faveur ${ }^{33}$.

29. Voir à ce sujet: A POPOVICI, «Le "locus delicti" en droit international privé québécois» (1982-83) 17 R.J.T. 463.

30. Moran c. Pyle National (Canada) Ltd, [1975] 1 R.C.S. 393 (appel de la Saskatchewan); voir aussi: Interprovincial Co-operatives Ltd et al. c. The Queen, [1976] 1 R.C.S. 477 (appel du Manitoba).

31. Voir CASTEL, op. cit., supra, note 2, p. 467; voir A. POPOVICI, loc. cit., stpra, note 29.

32. J.E. 84-19, C.S. Montréal, no 500-05-011811-834, 24 nov. 1983; (1982-83) 17 R.J.T. 463, commenté par A. POPOVICI, id., p. 463 et suiv.

33. POPOVICI, id, p. 469-473. 
D'abord c'était la solution de l'Ancien droit. Mais surtout, le dommage n'est source de l'obligation de réparer que parce qu'il résulte d'une violation d'une obligation légale (celle de s'abstenir de porter préjudice à autrui par intention malveillante ou par négligence). Comme le Québec ne pourrait constitutionnellement pas légiférer extra-territorialement, c'est-à-dire relativement à la commission d'un acte dommageable commis en dehors de son territoire, le droit québécois ne pourrait pas considérer comme fautif un tel acte ${ }^{34}$ s'il était valide selon la loi du lieu de commission.

L'auteur reconnaît toutefois que l'argument du droit constitutionnel devrait être examiné plus à fond ${ }^{35}$. Effectivement, une province peut exceptionnellement appliquer l'une de ses lois sans violer la constitution, même si ses dispositions affectent des droits «situés» en dehors de son territoire géographique, si ces effets sont incidents à l'exécution des pouvoirs de la législature ${ }^{36}$.

D'autre part, alors que la responsabilité fondée sur l'article 1053 C.c. nécessite l'existence d'une faute ou d'une négligence pour qu'il en découle une obligation de réparer le dommage, comme le rappelle l'auteur ${ }^{37}$, il existe des cas de responsabilité sans faute.

Or la responsabilité du constructeur, non fondée sur l'article 1053 C.c. mais sur l'article 1688 C.c., envisage plutôt le risque comme base légale, et constitue peut-être même une garantie de réparation du préjudice résultant du

\footnotetext{
34. Id, p. $469-471$.

35. Id, p. 471.

36. Voir en ce sens Ladore c. Bennett, [1939] A.C. 468 (P.C.), [1939] 2 W.W.R. 566 (P.C.); La Reine c. Thomas Equipment Ltd, [1979] 2 R.C.S. 529, [1979] 96 D.L.R. (3d) 1, 26 N.R. 499; Churchill Falls (Labrador) Corp. Ltd v. A. -G. of Newfoundland et al., (1984) 8 D.L.R. (4th) 1 (C.S.C.); [1984] 1 R.C.S. 297, 47 Nfld \& P.E.I.R. 125, 53 N.R. 268; British Airways 'Board v. Workers' Compensation Board, (1985) 17 D.L.R. (4th) 36 (B.C.C.A.); Re It's Adult Video Plus Ltd and McCausland, (1991) 81 D.L.R. (4th) 436 (B.C.S.C.). Voir à ce sujet: E. EDINGER «Territorial Limitations on Provincial Powers», (1982) 14 Ottawa L. Rev. 57, p. 71; J. BLOM, «The Conflict of Laws and the Constitution - Interprovincial Co-operatives Ltd v. The Queen", (1977) 11 U.B.C.L. Rev. 144, p. 154 et suiv.

37. Voir POPOVICI, loc. cit., supra, note 29, p. 472.
} 
vice de construction. Même si la faute reste l'impératif catégorique de la responsabilité civile ${ }^{38}$, il est très théorique de rattacher, pour les fins du droit international privé du moins, cette responsabilité pour vice de construction au lieu de violation de cet impératif catégorique...à moins de pouvoir situer dans l'espace cet impératif. En matière de responsabilité du constructeur, nous avouons donc ne pas pouvoir partager ce point de vue. Nous estimons que l'esprit du droit civil est respecté si l'on considère, au moins aux fins de la détermination de la loi applicable en droit international privé, que le préjudice subi du fait du vice de construction puisse constituer le fait localisateur de responsabilité.

De toute façon, le problème de la violation de l'impératif catégorique ne mène pas à autre chose qu'au dilemme devant lequel la Cour suprême du Canada s'est trouvée dans La Reine c. Thomas Equipment Ltd ${ }^{39}$ : s'il est artificiel de situer géographiquement un droit, une obligation, comment localiser une violation de cette obligation constituée par une faute d'abstention? Dans Thomas Equipment, il s'agissait de l'inexécution d'une obligation légale de reprendre des produits invendus édictée par une loi albertaine, par un fabricant du Nouveau-Brunswick, dont la loi, expressément choisie pour régir le contrat, ignorait une telle obligation. La Cour a estimé que le fabricant, en venant faire affaires en Alberta, s'était soumis à ce que l'on pouvait appeler l'impératif catégorique albertain.

Nous ne concevons pas de différence entre cette situation et celle du constructeur - ou fabricant - d'éléments préfabriqués en dehors du Québec, qui vient les installer ici, si la construction menace ensuite de s'écrouler. L'«abstention négligente» dure à partir de la fabrication jusqu'à l'intégration dans la construction définitive, au Québec, et ce fait réalise le vice de construction, entraînant le préjudice et l'obligation de réparation.

38. Voir POPOVICI, ibid, citant J.-L. BAUDOUIN, La responsabilité civile délictuelle, Montréal, Presses de l'Université de Montréal, 1973, p. 41, no 45.

39. Précité, supra, note 36. 
De toute façon, on peut soutenir que cette intégration constitue précisément le contact avec la sphère juridique québécoise ${ }^{40}$ - qu'on la considère comme fait générateur de préjudice ou non - donnant compétence constitutionnelle à la loi du Québec, selon les conceptions exprimées notamment dans les arrêts Ladore c. Bennett, La Reine c. Thomas Equipment Ltd ${ }^{41}$ et Churchill Falls (Labrador) Corp. Ltd v. A.-G. of Newfoundland.

En tout cas, toujours dans cette hypothèse de rattachement, si le préjudice était subi en dehors du Québec, c'est-à-dire au lieu, étranger, de la construction, il faudrait alors cumuler la loi étrangère et la loi québécoise. Il faut préciser à ce sujet que la simple violation d'un règlement étranger de construction, par exemple, suffirait pour remplir la branche lex loci delicti de la règle québécoise, même si cette violation n'entraînait à l'étranger qu'une sanction "pénale» ou administrative (amende). Par contre, la seule existence d'une telle sanction étrangère ne suffirait pas: il faudrait aussi vérifier que les faits, s'ils s'étaient déroulés au Québec, auraient pu justifier une action en responsabilité pour vice de construction. En définitive, on pourrait dire que l'utilisation de la règle de conflit «normale» dans notre domaine revient quasiment à appliquer la loi québécoise en tant que lex fori, en prenant en considération une situation à l'étranger - l'illégalité de l'acte - pour en tirer des conséquences en droit interne ${ }^{42}$.

40. Le professeur Popovici reconnaît volontiers la pertinence de l'argument (loc. cit., supra, note 29 , p. 472-473).

41. Arrêts précités, supra, note 36.

42. Pour cette notion de prise en considération, voir (en général) P. MAYER: «Formellement, la prise en considération se distingue de l'application en ce que la règle appliquée fournit elle-même la solution substantielle de la question posée tandis que la règle prise en considération est intégrée dans le présupposé de la règle qui se réfère à elle, et dont l'effet juridique détermine seul la substance de la relation juridique [...] la norme étrangère n'est qu'un élément du calcul intégré par la loi [du for].» («Droit international privé et droit international public sous l'angle de la notion de compétence", (1979) 68 Rev. crit. dr. internat. privé 1,349 , p. 368 , no 49); Voir dans le même sens en matière quasi délictuelle: The «Halley», (1868) L.R.2 P.C. 193, p. 203-204, repris dans Going v. Reid Brothers Motor Sales Ltd et al., (1982) 136 D.L.R. (3d) 254 (Ont. H.C.J.), p. 263: «The courts will, however, take into account foreigh rules of conduct to determine whether the tort is actionable by the lex fori. The rules, such as the speed limit [...] are incorporated into the domestic cause of action as facts to which the lex fori is applied" (nos italiques); voir cependant POPOVICI, loc. cit., supra, note 29, p. 471. 
Une seconde solution au problème du facteur de rattachement consiste à choisir un facteur propre, en suivant la tendance générale à l'éclatement de la règle de conflit extracontractuelle en fonction des différents types de responsabilité. Cette optique pourrait se fonder sur la nature quasi-contractuelle ou «purement» légale de cette responsabilité.

\section{ii. Choix d'un rattachement spécifique}

En assimilant la responsabilité du constructeur pour vice de construction à une question quasi contractuelle, comme une sorte d'enrichissement sans cause de sa part, on devrait appliquer la loi du «lieu de survenance du fait dont l'obligation résulte». On s'inspire ici de la formule peu compromettante

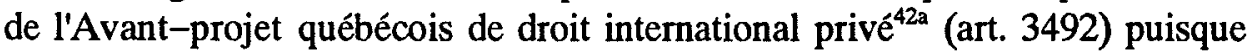
la jurisprudence n'a pas encore précisé le contenu de la règle de conflit ${ }^{43}$. Cette solution, qui revient exactement à reformuler, d'une autre manière, le problème de la recherche du fait générateur de responsabilité, aboutirait probablement, à notre avis, à la désignation de la loi du lieu de construction, puisque l'obligation résulte du vice de cette construction.

De la même façon, si l'on considère qu'il s'agit d'une responsabilité extracontractuelle «purement» légale, et non quasi délictuelle, on pourrait écarter la double règle jurisprudentielle propre à cette matière: on reviendrait alors à l'article 6 al. 3 C.c., qui permettrait de remonter au grand principe général de la territorialité qu'il exprime implicitement ${ }^{44}$ pour déterminer le facteur de rattachement - de type savignien - approprié. Cet article énonce en effet:

42a. Loi portant réforme au Code civil du Québec du droit de la preuve et de la prescription et du droit international privé, Assemblée nationale, $33^{\mathrm{inc}}$ Législature, $2^{\mathrm{ime}}$ session, Éditeur officiel du Québec, 1988. À ce sujet, voir notamment: J.A. TALPIS et G. GOLDSTEN, loc. cit., supra, note 8; A. PRUJINER, «Le statut des obligations en droit international privé, (1988) 29 C. de D. 1097-1127.

43. Voir en ce sens CASTEL, op. cit., supra, note 1, p. 489; GROFFIER, Précis, op. cit., supra, note 2, no 233. Voir aussi Y. LOUSSOUARN et P. BOUREL, Droit international privé, 3e éd., Paris, Dalloz, 1988, no 410, p. 643, cités par GROFFIER, no 233, p. 226.

44. Voir CRÉPEAU, loc. cit., supra, note 24, p. 15. 
«Les lois du Bas Canada relatives aux personnes sont applicables à tous ceux qui s'y trouvent, même à ceux qui n'y sont pas domiciliés; $[\ldots] »$.

Par ailleurs, cette qualification pourrait s'inspirer de l'arrêt Thomas Equipment ${ }^{45}$.

À notre avis, la solution reviendrait, là aussi, à appliquer la loi du lieu de construction, cette construction constituant le fait juridique le plus pertinent du point de vue du principe de territorialité: la conclusion du contrat au Québec ne devrait pas suffire.

Les conséquences et les problèmes des deux thèses ayant été envisagés, il faut déterminer quelle qualification, responsabilité civile contractuelle ou extracontractuelle, correspond le mieux à l'esprit du droit québécois (qualification «naturelle») ou à la fonction que les dispositions relatives à cette responsabilité particulière sont destinées à remplir (qualification dite «fonctionnelle»).

45. La Reine c. Thomas Equipment Ltd, précité, supra, note 36, p. 13 (D.L.R.) par le juge Martland: «The present case is not concerned with the contractual rights and obligations of the parties inter se. This case arises as a result of the imposition of statutory obligations upon vendors of farm implements who sell such machinery in Alberta. The agreement in question here involved the delivery of farm implements by a vendor to a dealer in Alberta for resale there and, in consequence, Thomas became subject to the duties imposed by the statute irrespective of what might be the proper law of the contract which, apart from the statute, would govern the rights of the parties inter se». Voir H.P. GLENN, «Conflict of Laws - Eviction of Proper Law of Contract by Legislation Creating Provincial Offence - Extraterritorial Effect of Provincial Legislation - Where is an Omission?»; (1981) $59 R$. du B. Can 840; R.K. PATERSON, «Do Unto Others: the Extraterritorial Reach of Regulatory Legislation in Canada», (1980-81) 5 Can. Bus. L.J. 114; J. BLOM, «Case Comments. Conflict of Laws and Constitutional Law - Extraterritorial Provincial Legislation - The Queen v. Thomas Equipment Ltd", (1982) 16 U.B.C.L. Rev. 357; EDINGER, loc. cit., supra, note 36; GROFFIER, Précis, op. cit., supra, note 2 , no 31 et 35 . 


\section{c. La qualification en droit positif interne}

On constate que la qualification extracontractuelle, légale, semble assez nettement l'emporter sur la qualification contractuelle en droit québécois.

À ce sujet, l'article 35 de la Loi québécoise sur la qualification professionnelle des entrepreneurs de construction ${ }^{46}$ dispose:

«La personne dont le principal établissement est situé hors du Québec et qui désire exécuter ou faire exécuter des travaux de construction au Québec doit obtenir de la Régie une licence temporaire délivrée selon les règlements et lui permettant d'exécuter ou de faire exécuter ces travaux.

Pour obtenir une licence, son maintien ou son renouvellement, le demandeur doit élire domicile au Québec. [...]»

Cet article définit son domaine à la fois par rapport au domicile du constructeur et au lieu d'exécution des travaux, mais il est pourtant clair que l'élément essentiel qui déclenche l'application de ce régime (incluant l'obligation d'élire un domicile au Québec et de remettre un cautionnement) est le fait de construire au Québec. Nous avons donc ici un fort indice de la conception que retient le législateur québécois de la territorialité dans notre domaine, au sujet d'une loi de droit public, imposant des contraintes ou des obligations légales.

Il est essentiel de bien saisir le sens que le législateur a donné à cette disposition, car elle constitue pratiquement la seule esquisse d'une réglementation en droit international privé des contrats de construction et de la responsabiliré civile du constructeur.

L'article 35 est à notre avis la charnière entre les deux éléments dont le législateur québécois disposait. D'un côté, comme nous allons le préciser plus loin, la jurisprudence de droit interne s'orientait assez nettement vers une qualification extracontractuelle de la responsabilité du constructeur pour vice

46. L.R.Q. 1977 , c. Q-1. 
de construction, et semblait donc empêcher l'utilisation de la règle de conflit contractuelle.

De l'autre, l'article 6 al. 3 C.c., clef de voûte de la responsabilité extracontractuelle en droit international privé québécois, semble exiger la «présence» au Québec des personnes visées. Or le fondement de la responsabilité de l'article 1688 C.c. est soit le fait générateur du préjudice, c'est-à-dire une faute (d'abstention) de la part du constructeur - l'acte dommageable soit le préjudice subi, c'est-à-dire le risque que l'écroulement de la construction entraîne pour le maître d'ouvrage et surtout pour le public en général.

Donc, pour respecter à la fois la lettre de l'article 6 al. 3 C.c. et la qualification jurisprudentielle de la responsabilité, et pour atteindre les buts de protection du public local québécois, la structure adoptée en conséquence fut l'exigence de cette élection de domicile au Québec pour tout constructeur étranger qui voulait exécuter des travaux de construction sans $s^{\prime} y$ trouver d'une façon stable. Ainsi, on s'assurait que ce constructeur se trouvait au Québec et que sa responsabilité pour vice de construction en cette province, découlant de l'article 1688 C.c., pouvait être directement fondée sur l'article 6 al. 3 C.c.

À notre avis, c'est précisément de cette exigence d'une élection de domicile au Québec que l'on peut tirer une preuve tangible de l'intention du législateur québécois de choisir la règle de conflit extracontractuelle pour régir la responsabilité découlant de l'article 1688 C.c. La formulation unilatéraliste de la règle de conflit implicitement consacrée se prêtait d'ailleurs à une bilatéralisation, mais le législateur n'était pas préoccupé par l'écroulement des constructions situées hors du Québec.

Comme nous l'avons dit, la conception territorialiste de l'article 35 de la Loi sur la qualification professionnelle des entrepreneurs de construction, correspond par ailleurs à la qualification que semble favoriser la jurisprudence québécoise en droit interne. Dans l'affaire La Fabrique de la Paroisse de St-Philippe d'Arvida c. Desgagné ${ }^{-47}$, la Cour d'appel affirme:

47. [1979] C.A. 198. 
«L'article 1688 C.C. crée un régime spécial de responsabilité, soit une responsabilité légale dont le cadre, d'une part, coïncide pour partie avec celui de la responsabilité du droit commun, soit la responsabilité contractuelle, et, d'autre part, le déborde substantiellement. ${ }^{48}$ "

Selon cette opinion, il s'agirait donc d'une responsabilité légale dont le fait générateur serait le vice de construction, même si elle recouvre des situations sujettes, par ailleurs, à la responsabilité contractuelle ${ }^{49}$. Cette superposition de domaines ne rendrait pas contractuelle cette obligation, que d'aucuns considèrent comme de garantie. Ainsi M. le juge Bélanger, dans la même affaire, affirme:

«À mon avis, c'est une garantie que crée l'article 1688 C.C.; le fondement juridique du recours est un fait: le vice de la construction ou du sol mettant l'édifice en péril. Ce recours n'épuise pas tous les droits du propriétaire, ceux dont le fondement est la faute contractuelle qu'il doit prouver. ${ }^{50}{ }_{\gg}$

M. le juge Bernier soutient de son côté:

«[L'article 1688] vise une situation de fait bien précise: celle de la perte partielle ou totale de l'édifice par suite d'un vice de construction ou du sol. Dans ce cas où la responsabilité de l'architecte ou de l'entrepreneur ne serait normalement engagée en vertu du droit commun que si le maître de l'oeuvre prouve un défaut ou vice contractuel de leur part, l'article 1688 C.C. impose [...] une respon-

48. Id, p. 204-205.

49. Au Québec, l'article 1688 C.c. incorpore au Code civil l'arrêt Brown c. Laurie, ((1855) 5 L.C.R. 65 B.R.), repris, par le Conseil privê dans Wardle c. Bethune, (1871) L.R.4 (P.C.) Ap. 33 où il est dit, p. 54-55: «there is annexed to the contract, by force of law, a warranty of the solidity of the building..." (nos italiques). La Cour suprême du Canada a notamment suivi cet arrêt dans Canadian Consolidated Rubber Co. c. T. Pringle \& Son Ltd and The Foundation Co. Ltd, [1930] R.C.S. 477, où il est dit à la p. 480: «According to the decision of the Privy Council referred to, article 1688 of the Civil Code imported into the contracts [...] a warranty of the stability of the dam for ten years». (nos italiques)

50. La Fabrique de la Paroisse de St-Philippe d'Arvida c. Desgagné, précité, supra, note 47, p. 202-203 (nos italiques). 
sabilité légale conjointe et solidaire dont seuls le cas fortuit ou la faute entière du maître de l'oeuvre ou d'un tiers pourront les relever; la loi les rend garants de la solidité de l'oeuvre; $[\ldots]^{51} \gg$

À l'encontre de cette qualification, on peut opposer qu'en réalité, même si la loi crée cette obligation de réparation, elle ne le fait qu'en raison de l'existence d'un contrat et elle l'intègre d'ailleurs dans le champ contractuel ${ }^{52}$; de toute façon, la qualification d'obligation de «garantie» n'exclut pas cette responsabilité du domaine contractuel.

Par ailleurs le fait qu'elle soit généralement considérée d'ordre public $^{53}$ - au moins en droit interne - ne permet pas non plus de trancher la question. Au contraire, en faveur de la qualification contractuelle, on peut invoquer le fait que, bien qu'il n'existe évidemment aucun contrat entre l'acheteur et le constructeur ou l'architecte, le droit - de source contractuelle aux dommages, en vertu de l'article 1688 C.c. est transmis aux acheteurs successifs de l'édifice, à titre d'accessoire du droit de propriétét ${ }^{54}$.

La qualification s'effectuant, nous l'avons dit, selon les catégories élargies - du droit civil interne du for, il nous appartient, dans le cadre de cet article portant sur le droit international privé, d'essayer de déterminer la qualification qui correspond le mieux à l'esprit du droit civil, mais il n'est pas dans notre intention de régler ici la controverse en droit civil interne. Or, la

51. Id, p. 205. Nous ne partageons pas totalement ce point de vue: s'il s'agit vraiment d'une obligation de garantie, le cas fortuit ne doit pas libérer le garant de sa responsabilité.

52. ROUSSEAU-HOULE, Les contrats de construction en droit public et privé, Montréal, Wilson \& Lafleur, Sorej, 1982, p. 310-311.

53. La Fabrique de la Paroisse de St-Philippe d'Arvida c. Desgagné, précité, supra, note 47, p. 205 par le juge Bernier: «[I]l s'agit d'une disposition d'ordre public que les parties par convention ne pourront à l'avance même minimiser». Voir aussi Davie Shipbuilding Ltd c. Cargill Grain Co. Ltd, [1978] 1 R.C.S. 570 (cité par le juge, ibid.).

54. . La Fabrique de la Paroisse de St-Philippe d'Arvida c. Desgagné, précité, supra, note 53, p. 205; McGuire c. Fraser, (1908) 17 B.R. 449 conf. par (1908) 40 R.C.S. 577 (cité par le juge, ibid.). 
doctrine - notamment la doctrine française - est profondément divisée à ce sujet $^{55}$.

À notre connaissance, la seule décison qui traite de cette question de qualification, en droit international privé, est l'arrêt de la Cour d'appel rendu en 1976 dans l'affaire Procon (Great Britain) Ltd v. Golden Eagle Co. Ltd ${ }^{56}$. La Cour y écartait l'effet d'une clause compromissoire, contenue dans un contrat d'entreprise, donnant compétence à la Cour d'arbitrage de Londres, au motif que l'action, fondée sur l'article 1688 C.c., était une question de responsabilité légale et non une action fondée sur le contrat: la clause compromissoire était donc applicable.

La solution revenait à dire ce que nous soutenons: en droit québécois les parties n'ont pas le choix de la loi applicable à la responsabilité civile du constructeur fondée sur l'article 1688 C.c. parce que cette question ne relève pas du domaine de la loi d'autonomie. Toutefois, on peut expliquer cette solution de deux façons: les parties n'ont pas le choix, soit parce qu'il s'agit d'une question extracontractuelle et que la règle de conflit propre à ce domaine envisage l'application impérative de la loi territoriale (art. 6 al. 3 C.c.), soit parce que, même si l'on se trouve dans le domaine contractuel, la loi d'autonomie se heurte à certaines lois impératives, tel l'article 1688 C.c., comme le prévoit l'article 8 C.c.

Quoi qu'il en soit, en droit international privé québécois, nous estimons qu'actuellement les prétentions relatives à cette responsabilité pour vice de construction seraient probablement soumises à une règle de conflit extracontractuelle plutôt qu'à la loi d'autonomie. Il en résulterait que les parties ne pourraient pas choisir une autre loi que celle du lieu du fait générateur de l'obligation légale. De plus, à notre avis, cette loi territoriale devrait être celle

55. Voir à ce propos ROUSSEAU-HOULE, op. cit., supra, note 52, p. 310-314. L'auteur penche plutôt pour la qualification «légale», tout comme l'O.R.C.C. (id., p. 313). Elle cite des décisions allant dans les deux sens ou même qui semblent cumuler les qualifications (id., p. 313-315)

56. [1976] C.A. 565. Voir M. LALONDE, J.N. BUCHANAN, J.C. ROSS, «Domestic and International Commercial Arbitration in Quebec: Current Status and Perspectives for the Future», (1985) 45 R. du B. 705, p. 722-725. 
du lieu d'exécution des travaux - lex loci delicti - qui correspondrait aussi avec celle du lieu de situation de l'immeuble.

En tout cas, pour être pertinente, fonctionnelle, la formulation de la règle de conflit québécoise devrait tenir compte de la politique interne québécoise de protection du maitre d'ouvrage et du public. En restant dans la méthode traditionnelle et en admettant une qualification extracontractuelle, on est poussé à souhaiter l'adoption d'une règle de conflit à caractère substantie$1^{56 \mathrm{a}}$ comportant un facteur de rattachement alternatif. On pourrait simplement adapter la règle de conflit relative à la responsabilité du fabricant de bien mobilier, dans le futur Code civil. L'article 3128 du Projet de loi 125 donne le choix à la victime entre la loi du lieu de résidence du fabricant et celle du lieu d'acquisition du bien ${ }^{57}$. Notre règle de conflit se traduirait ainsi par un choix possible, selon la loi la plus favorable au maître d'ouvrage, entre celle de l'établissement principal du constructeur et celle du lieu de la construction. De cette façon, on prolongerait au plan international la politique destinée à protéger le public puisque le constructeur serait tenu aux standards de la loi la plus sévère. De plus, cette loi correspondrait sans doute aux attentes des parties: le constructeur connaît la loi de son établissement principal et ne peut prétendre être surpris par l'application de celle du lieu étranger où il va construire. Il faut toutefois noter que l'article 3127 du même projet impose l'application de la loi contractuelle, à l'exclusion de la loi extracontractuelle, «[1]orsque l'obligation de réparer un préjudice résulte de l'inexécution d'une obligation contractuelle». En matière de responsabilité du constructeur pour vice de construction, si l'on soutient que l'obligation de réparer le préjudice résulte du fait du vice de construction et non du contrat, cette disposition ne devrait pas écarter l'application de la loi extracontractuelle.

56a. Voir à ce sujet, notamment: P.M. PATOCCHI, Règles de rattachement localisatrices et règles de rattachement à caractère substantiel, Genève, Georg, 1985; A. BUCHER, «Sur les règles de rattachement à caractère substantiel», dans Liber Amicorum Adolf $F$. Schnitzer, Genève, Georg, 1979, p. 37.

57. Précité, supra, note 6, art. 3128: «La responsabilité du fabricant d'un bien meuble, quelle qu'en soit la source, est régie, au choix de la victime: $1^{\circ}$ Par la loi de l'État dans lequel le fabricant a son établissement ou, à défaut, sa résidence; $2^{\circ} \mathrm{Par}$ la loi de l'État dans lequel le bien a été acquis». 
Toutefois, si les juges acceptaient une qualification contractuelle, les parties seraient libres de choisir une loi étrangère mais la législation québécoise pourrait et devrait intervenir comme loi de police ou au nom de l'exception d'ordre public. Avant d'envisager cette possibilité, on doit, dans les deux cas, déterminer le domaine de la loi applicable à la responsabilité civile du constructeur.

\section{Le domaine de la loi applicable à la responsabilité civile du construc- teur}

Ce domaine devrait comprendre nécessairement des questions comme la validité des clauses d'exclusion de responsabilitét ${ }^{58}$, la détermination des personnes qui y sont soumises et ses conditions, notamment le problème de savoir si le fait générateur doit avoir ou non le caractère d'une faute ${ }^{59}$, ses causes éventuelles d'exonération, l'étendue de la solidarité qui en résulte et la durée de cette responsabilité ( 5 ou 10 ans), etc.

Mais quelle loi gouverne les délais d'action en responsabilité, prévus au Québec dans l'article 2259 C.c.? Il ne s'agit plus ici du principe de la responsabilité, ni de son contenu, mais plutôt d'une question de prescription de l'action, ou de «délai préfix» ${ }^{60}$. Le choix qui se présente ici consiste soit, comme on le fait normalement en droit civil, à faire régir la prescription par la loi applicable au fond - ici, la loi gouvernant la responsabilité civile du constructeur - soit, comme en common law, à la soumettre à la loi du tribunal saisi en l'assimilant à la procédure. Il n'existe malheureusement pas au Québec

58. Voir en ce sens, au sujet de la vente, le récent arrêt de la Cour de Cassation ( $1^{\text {ire }}$ Ch. civ.), 4 octobre 1989, (Soc. De Baat en Zegwaard c. Soc. les Fils Charvet et Porteix), (1990) 79 Rev. crit. dr. internat. privé 316, note P. LAGARDE.

59. Voir en ce sens A. TOUBIANA. Le domaine de la loi du contrat en droit international privé: Contrats internationaux et dirigisme étatique, Paris, Dalloz, 1972, no 143, p. 117.

60. Sur la prescription en droit international privé, voir notamment F. HAGE-CHAHINE, Les conflits dans l'espace et dans le temps en matière de prescription, Paris, Dalloz, 1977; «La vérité jurisprudentielle sur la loi applicable à la prescription extinctive de l'obligation», dans Mélanges Weill, Paris, Dalloz et Litec, 1983, p. 303. 
de règle générale de conflit relative à la prescription ${ }^{61}$, à l'exception de l'article 2189 C.c., soumettant la prescription immobilière à la lex rei sitae ${ }^{62}$.

La question est rendue très complexe en raison de l'existence de véritables règles matérielles à but international d'une rédaction plutôt obscure, contenues aux articles 2190 et 2191 C.c. ${ }^{63}$. Elles déterminent les modalités de calcul de prescription (jonction de périodes etc.) acquise selon la loi québécoise ou étrangère, mais ne précisent pas en vertu de quelle loi leur prise

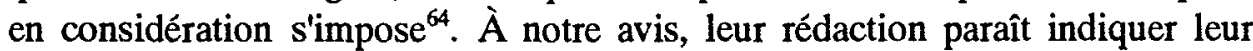
rattachement à la procédure: d'une part, les facteurs déterminant leurs règles de calcul sont en fait des chefs de compétence juridictionnelle et d'autre part, elles restent muettes quant à la loi applicable à la cause du recours à la prescription, ce qui suggère à ce sujet l'application de la loi du for.

61. Voir à ce sujet: J.-G. FRÉCHETTE, «La prescription en droit international privé», (1972) 3 R.D.U.S. 121; CASTEL, op. cit., supra, note 2, p. 744 et suiv.; GROFFIER, Précis, op. cit., supra, note 2, no 236, p. 228.

62. Cette règle ne s'applique pas à la responsabilité pour vice de construction, même d'un immeuble, parce que cette question ne le touche qu'indirectement. Il s'agit essentiellement de déterminer la loi applicable à la durée d'une action personnelle.

63. «Art. 2190. En matière de biens meubles et d'actions personnelles, même en matière de lettres de change et de billets promissoires, et en affaires de commerce en général, l'on peut invoquer sếparément ou cumulativement:

1. La prescription entièrement acquise sous une loi différente lorsque la cause d'action n'a pas pris naissance dans le Bas Canada, ou que la dette n'y a pas été stipulée payable, et lorsque cette prescription a été ainsi acquise avant que le possesseur ou le débiteur y ait eu son domicile;

2. La prescription entièrement acquise dans le Bas Canada, à compter de l'échéance de l'obligation, lorsque la cause d'action y a pris naissance ou que la dette y a été stipulée payable, ou que le débiteur y avait son domicile à l'époque de cette échéance; et dans les autres cas à compter de l'acquisition de ce domicile par le débiteur ou le possesseur;

3. La prescription résultant de temps successifs écoulés dans les cas de deux paragraphes précédents, lorsque le temps écoulé sous la loi différente a précédé.

Art. 2191. Les prescriptions qui ont commencé à courir sous l'empire des lois du Bas Canada son parachevées conformément aux mêmes lois, sans préjudice à invoquer celles qui s'étaient auparavant accomplies sous une loi différente, ou les temps combinés d'après l'une ou l'autre loi, conformément à l'article qui précède.

64. Voir en ce sens, GROFFIER, Précis, op. cit., supra, note 2, no 236, p. 228. 
En définitive, il semble bien que ces dispositions, tout comme la double règle jurisprudentielle applicable en matière de responsabilité quasi délictuelle, n'organisent en fait que la «prise en considération» de solutions découlant de l'applicabilité de lois étrangères au fond du litige, sans envisager leur application aux questions de prescription qui en découlent ${ }^{65}$.

La jurisprudence, en tout cas, résoud les problèmes de prescription sans aucune constance, certains arrêts utilisant les règles de la loi du for (québécoise), d'autres, les règles étrangères de prescription de la loi régissant au fond le rapport ayant donné naissance à l'action (lex causae) ${ }^{66}$. Cette dernière solution, celle du droit civil en général, apparaît plus satisfaisante aux auteurs québécois ${ }^{67}$, malgré les difficiles problèmes de preuve de la loi étrangère qu'elle comporte.

Sans prendre parti dans ce débat difficile, on peut tenter d'examiner l'application des articles 2190 et 2191 C.c. dans quelques cas qui nous concernent directement.

Si la construction a lieu au Québec, la loi québécoise s'appliquera à la prescription, ou à la durée du droit d'action, relative à la responsabilité du constructeur pour vice de construction, en tant que loi du for (applicable à la procédure) ou comme lex causae (applicable en tant que loi du lieu d'exécution de la construction - loi territoriale). Alors l'article 2190.2 permettra d'invoquer la prescription entièrement acquise selon la loi québécoise puisque la «cause d'action» - l'un des critères retenus par cette disposition - y aura pris naissance.

Toujours dans la même hypothèse, si la prescription n'est pas totalement «acquise» selon la loi québécoise, c'est-à-dire si l'action contre le constructeur se situe par exemple à l'intérieur de la durée de 5 ans à partir de la perte (art. 2259 al. 1 C.c.), les articles 2190 et 2191 C.c. ne devraient pas

65. Voir à ce sujet, supra, note 41.

66. Voir sur cette jurisprudence, CASTEL, op. cit., supra, note 2, p. 745-747.

67. Voir en ce sens CASTEL, id, p. 748; FRÉCHETTE, loc. cit., supra, note 61, p. 126. 
permettre de faire appel à une prescription plus courte découlant d'une loi étrangère, par exemple de celle de l'établissement principal de l'entrepreneur.

Si la construction n'a pas lieu au Québec, la loi québécoise n'est plus la lex causae mais uniquement la loi du for ${ }^{68}$. En réalité, peu importe alors, pour appliquer les articles 2190 et 2191 C.c., le rattachement choisi pour la prescription. Lex causae et lex fori peuvent éventuellement cumuler leurs années de prescription pour dégager le constructeur de la responsabilité pour vice de construction (art. 2191 C.c. et art. 2190.3 C.c.). Sans cumul, il sera possible par exemple d'invoquer le fait que le recours est prescrit selon la loi étrangère du lieu de la construction, à condition que cette prescription ait été entièrement acquise avant que le constructeur n'ait acquis un domicile québécois (art. 2190.1 C.c.).

Si le recours en responsabilité pour vice de construction n'est pas encore prescrit selon la loi étrangère du lieu de construction lorsque le constructeur a acquis un domicile au Québec, celui-ci pourra invoquer la prescription du droit québécois (art. 2259 C.c.); il cumulera les années de prescription pendant lesquelles il n'était pas domicilié au Québec, avec celles écoulées sous la loi québécoise ${ }^{69}$.

Dans ce cas, on voit bien qu'il n'y a pas d'application de la loi étrangère, en tant que lex causae, à la prescription mais simple prise en

68. Voir par analogie, en matière de responsabilité quasi-délictuelle pour accident de la route: The Scottish Metropolitan Ass. Co: Ltd v. Graves, [1955] C.S. 88, p. 95; Beach v. Avis Transport of Canada Ltd et al., [1978] C.S. 418.

69. The Scottish Metropolitan Ass. Co. Ltd v. Graves, ibid. Un Arrêt (Holtzheuser et al. c. Robberecht, [1961] C.S. 423) a cependant appliqué une méthode de calcul proportionnel, proposée par Mignault (P.B. MIGNAULT, Droit civil canadien, t. 9, Montréal, Wilson \& Lafleur, 1916, p. 355-356). On ne cumule pas simplement les années de prescription à atteindre en tenant compte de deux lois. Mignault expliquait qu'autrement le calcul revenait purement et simplement à toujours appliquer «la prescription de notre droit, et alors, il était inutile de parler de la jonction des deux périodes", (id., p. 355).

Notre analyse est différente: la loi québécoise s'applique seule mais elle prévoit une règle matérielle spéciale à but international, qui prend en considération, sans l'appliquer en tant que telle, la loi étrangère et sa durée de prescription, pour en tirer des conséquences en droit québécois. 
considération d'années passées en regard d'une loi étrangère pour un calcul exclusivement fondé sur la loi québécoise (applicable en tant que loi procédurale du for). Mais quel système juridique étranger prend-on en considération? Les articles ne le disent pas, cependant la jurisprudence a précisé, en matière de quasi-délit, qu'il s'agissait de celui où la cause d'action a pris naissance ${ }^{70}$. On a ainsi pratiquement bilatéralisé l'article 2190 C.c. En tout cas les cours n'ont pas décidé pour autant que la loi étrangère s'appliquait en matière dé prescription, en tant que lex causae (ou loi applicable au fond du litige) $)^{71}$

Ayant examiné les questions posées par la détermination de la loi applicable à la responsabilité du constructeur, il reste à étudier l'hypothèse de la mise à l'écart de la loi étrangère désignée, au nom de l'ordre public québécois, ou encore celle de l'intervention de lois de police dans le domaine.

\section{L'ordre public international et la responsabilité civile du cons- tructeur pour vice de construction}

Nous traiterons cette question en deux points. Qu'elle se pose au sujet des lois de police ou par le biais de l'exception d'ordre public, elle comporte en effet deux aspects: les dispositions québécoises relatives à la responsabilité du constructeur pour vice de construction ou du sol sont-elles impératives en droit international privé? (A) Dans l'affirmative, dans quelles situations internationales interviendraient-elles? (B)

70. Ibid. Voir aussi Beach v. Avis Transport of Canada Ltd et al., précité, supra, note 68.

71. Beach v. Avis Transport of Canada Ltd et al., id, p. 421 par le juge Rothman: «But even if one were to accept the distinction between substantive and procedural questions in the present case, I am not aware of any Quebec authority to support the proposition that prescription is always to be governed by the law of the place where the cause of action arose because it is a substantive matter. There is, however, some authority to the contrary.» 
A. La responsabilité civile du constructeur pour vice de construction est-elle d'ordre public international?

Envisageons la situation où la construction est érigée à l'étranger. Le juge québécois, saisi par le maître d'ouvrage en raison du domicile du constructeur au Québec, donnerait-il gain de cause au premier s'il invoquait l'application des articles 1688 C.c. et 2259 C.c., alors que la loi étrangère ne comprend par hypothèse aucune responsabilité de ce type?

Dans l'optique d'une qualification extracontractuelle de la responsabilité du constructeur, on s'aperçoit qu'il y a assez peu de place pour un raisonnement en terme d'exception d'ordre public. En effet, si l'on prend pour acquis la double règle de common law s'appliquerait alors, nous avons vu que la jurisprudence a décidé qu'il suffit que l'acte soit «injustifiable» selon la lex loci delicti pour que la responsabilité de la loi québécoise, applicable en tant que lex fori, intervienne. Or, il serait assez difficile de trouver une loi étrangère qui justifierait le constructeur de bâtir une construction menaçant ruine, ou de ne pas prêter attention à l'état du sol pour construire, sans au moins prévoir une responsabilité contractuelle ou quasi délictuelle.

Si, au contraire, la responsabilité étrangère de la lex loci delicti était plus large que celle du droit québécois, alors cette dernière, lex fori, interviendrait de toute façon en vertu de la seconde «branche» de la règle québécoise, pour «tailler» la responsabilité à la mesure du droit québécois. En d'autres termes, la règle de conflit comprenant elle-même l'application de la loi du for, l'exception d'ordre public est exclue puisque celle-ci n'intervient que pour écarter la loi étrangère.

Si la règle de conflit était plutôt directement tirée du principe de territorialité (art. 6 al. 3 C.c.), toujours en considérant le vice de la construction comme le fait juridique justifiant la responsabilité, alors la question de l'intervention extraterritoriale des dispositions québécoises à l'encontre d'une loi étrangère pourrait se poser directement du fait de l'article 6 al. 3 si le constructeur avait un établissement au Québec ou indirectement au nom de l'ordre public si d'une façon ou d'une autre la solution étrangère choquait les conceptions québécoises. 
Mais c'est surtout dans l'hypothèse où l'on qualifierait la responsabilité de contractuelle que le problème deviendrait réel puisque les parties pourraient écarter le droit québécois, notamment l'article 1688 C.c., même au sujet d'une construction érigée au Québec. On devrait alors faire appel à l'exception d'ordre public pour éviter ce résultat.

D'ailleurs, dans cette optique, il ne faudrait qu'un pas pour franchir le Rubicon méthodologique ${ }^{72}$ et considérer l'article 1688 C.c. comme une loi de police, à partir du moment où le caractère impératif de cette disposition apparaîtrait suffisamment vital pour le Québec.

Il faut donc d'abord se demander si cette disposition a un caractère suffisamment impératif, en droit interne, pour «forcer la compétence» internationale de l'ordre juridique québécois, directement (en tant loi de police) ou indirectement (par l'exception d'ordre public).

$\mathrm{Au}$ Québec comme ailleurs, on fonde cette responsabilité civile sur deux motifs, pour la considérer d'ordre public interne: l'ignorance du maître d'ouvrage, face à l'expertise du constructeur (motif d'intérêt privé) et la protection de la sécurité du public vis-à-vis de bâtiments dangereux (motif d'intérêt public) ${ }^{73}$.

En définitive, seul le second constituerait éventuellement un motif súffisant pour forcer la compétence de l'ordre juridique local québécois. Il faut préciser qu'en droit interne, le maitre d'ouvrage, s'il est lui-même expert, ne bénéficie pas de la protection de l'article 1688 C.c. $^{74}$, ni lorsqu'il intervient dans la construction ${ }^{75}$. De plus, la majorité de la jurisprudence affirme qu'il

72. Voir, en faveur d'une telle approche, en général: J. TALPIS, loc. cit., supra, note 28; voir aussi GROFFIER, Précis, op. cit., supra, note 2, no 31 et 32, p. 35-37.

73. Voir J.W. DURNFORD, «The Liability of the Builder, Architect and Engineer for Perishing and Other Defects in Construction", (1967) 2 R.J.T. 161, p. 184; ROUSSEAUHOULE, op. cit., supra, note 52, p. 315 et suiv.

74. Davie Shipbuilding Ltd c. Cargill Grain Co. Ltd, précité, supra, note 53; ROUSSEAUHOULE, id, p. 316-317.

75. DURNFORD, loc. cit., supra, note 73, p. 186; Davie Shipbuilding Ltd c. Cargill Grain Co. Ltd, ibid; contra, ROUSSEAU-HOULE, id., p. 316. 
est illégal de se dégager à l'avance d'une telle responsabilité par une clause contractuelle, bien qu'un juge se soit récemment déclaré prêt, en obiter, à accepter l'exclusion de garantie par une clause expresse sans ambiguïté ${ }^{76}$.

Dans tous ces cas, que devient l'intérêt public? Le problème est bien là: la politique de protection préventive des tiers ne peut être toujours maintenue par ce moyen indirect précisément en raison du cadre contractuel. Cette «annexe» contractuelle ne peut normalement pas être invoquée par les tiers, peut être insuffisamment protégés par la responsabilité quasi délictuelle de droit commun. La fiction atteint ses limites.

Dans ces circonstances, il ne nous semble pas évident de pouvoir tirer de cette législation un principe justifiant l'intervention de la loi québécoise à titre d'exception d'ordre public, pour écarter la solution matérielle de la loi étrangère ${ }^{77}$. A fortiori, le même raisonnement peut être étendu à l'analyse en terme de loi de police.

On doit toutefois rappeler que la Cour d'appel en 1976, dans l'affaire Procon (Great Britain) Ltd v. Golden Eagle Co. Ltd ${ }^{78}$ a écarté une clause compromissoire contenue dans un contrat d'entreprise, en vertu de l'ancien article 940 C.p.c. qui prescrivait qu'on ne pouvait compromettre sur les questions concernant l'ordre public ${ }^{79}$. Les motifs étaient que l'action fondée sur l'article 1688 C.c. était une question de responsabilité légale et non une action fondée sur le contrat - donc que la clause était inapplicable. De plus, l'article 1688 C.c. serait une disposition d'ordre public ${ }^{80}$, donc non susceptible de compromis.

76. Sergerie c. Houde, J.E. 79-439, C.S. Terrebonne, no 05-002011-769, 2 avril 1979, p. 12-13 du jugement.

77. Voir dans le même sens: DESCHAMPS, loc. cit., supra, note 1, p. 479.

78. Précité, supra, note 56.

79. Voir maintenant l'article 1926.2 C.c. du même effet. Mais voir pour une critique de sa formulation: G. GOLDSTEIN et J. TALPIS, L'effet au Québec des jugements étrangers en matière de droits patrimoniaux, Montréal, Thémis, 1991, p. 243-244.

80. GOLDSTEIN et TALPIS, id, 567. 
Par conséquent, il faut déterminer les cas où l'on pourrait estimer nécessaire que la loi québécoise intervienne.

\section{B. Le domaine des articles 1688 et 2259 C.c. s'ils étaient considérés d'ordre public international}

Que l'analyse soit effectuée en terme d'exception d'ordre public ou en terme de loi de police, il faut déterminer à quelles situations internationales ces dispositions s'appliqueraient. Ceci est bien évident pour la loi de police. Mais, de même, l'exception d'ordre public ne doit intervenir que si la solution matérielle donnée par la loi étrangère heurte l'ordre public du for ${ }^{81}$.

Cette conception classique nécessite, à notre avis, la présence d'un impact, normalement de nature territoriale, sur l'ordre juridique québécois ${ }^{82}$. Or, la politique québécoise de protection du public, à la base du caractère d'ordre public de la responsabilité du constructeur, ne nous semble raisonnablement concerner que la population québécoise dans son ensemble.

On n'imaginerait pas en effet le législateur québécois envisager, par cette responsabilité, la protection préventive des Québécois quel que soit l'endroit où l'un d'entre eux se trouverait. On pourrait dire, si l'on nous pardonne cet emprunt à la théorie américaine des intérêts gouvernementaux, que le Québec n'aurait aucun intérêt légitime à mettre en oeuvre, de façon universelle, sa politique préventive sous-tendant l'article 1688 C.c.

En premier lieu, dans l'hypothèse d'une qualification extracontractuelle de cette responsabilité, aucune loi étrangère ne pourrait avoir cet impact nécessaire sur l'ordre juridique québécois si la loi locale québécoise régit exclusivement la responsabilité du constructeur vis-à-vis des constructions érigées au Québec, les seules qui menacent la sécurité de l'ensemble de la société québécoise.

81. Voir P. LAGARDE, Recherches sur l'ordre public en droit international privé, Paris, L.G.D.J. 1959, no 140 , p. 164-165.

82. Nous faisons référence ici à la théorie, connue notamment en Suisse et en Allemagne, et récemment assez utilisée dans la jurisprudence française, de la Binnenbeziehung (voir notamment, LAGARDE, id, no 48 et suiv., p. 55 et suiv.). 
De la même façon, si l'on envisage maintenant la question sous l'angle des lois de police, le seul intérêt vital que le législateur québécois puisse, à notre avis, exprimer dans notre domaine, ne concerne que la sécurité des Québécois vis-à-vis des édifices au Québec ${ }^{83}$. Comme la règle de conflit régissant la responsabilité extracontractuelle (confondue à l'origine avec les «lois de police ou de sûretét ${ }^{84} \gg$ ) est tirée elle-même du principe de territorialité, il n'est pas nécessaire de faire appel à la notion de loi de police à ce sujet, dans cette hypothèse de qualification.

Par conséquent, si la responsabilité spéciale des constructeurs était considérée d'ordre public international au Québec, ni l'exception d'ordre public, ni la théorie des lois de police ne justifieraient, à notre avis, d'attribuer aux dispositions québécoises un domaine d'application international différent de celui résultant de la règle de conflit extracontractuelle. Ceci milite, à notre sens, pour une telle qualification, au moins pour les fins du droit international privé, puisqu'on éviterait ainsi de faire appel à des moyens dont on s'entend à reconnaître que le domaine doit être le plus restreint possible.

Si l'on envisage au contraire la seconde hypothèse de qualification responsabilité contractuelle - la loi québécoise pourrait, et même devrait être invoquée au nom de l'exception d'ordre public malgré un choix exprès ou même implicite en faveur d'une loi étrangère, toutes les fois que la construction viciée menacerait de s'écrouler au Québec; cet impact serait suffisant pour justifier cette intervention. De fait, il est impensable de ne pas se plier aux exigences impératives de la loi du lieu de construction.

Par contre, on pourrait difficilement considérer comme légitime ce raisonnement si un maître d'ouvrage ou un acheteur postérieur, domicilié au Québec, subissait un préjudice du fait d'une construction viciée élevée en dehors de cette province, et que la loi étrangère applicable ne réparerait pas de la même façon que l'article 1688 C.c. En ce cas, seul l'intérêt privé

83. Voir l'article 35 de la Loi sur la qualification professionnelle des entrepreneurs de construction, précitée, supra, note 46.

84. Voir à ce sujet: T. VANDER ELST, Les lois de police et de sûreté en droit international privé français et belge, Bruxelles, Parthénon, t. 1, 1956, t. $2,1963$. 
subsisterait et l'attribution du caractère impératif, d'ordre public, de la responsabilité serait difficile à justifier ${ }^{85}$.

Enfin, si l'on estimait devoir faire de cet article 1688 C.c. une loi de police, pour les mêmes raisons, de toute façon, son domaine d'application ne devrait pas dépasser les limites du territoire.

Pour conclure sur ce point, nous avouons franchement notre préférence, aux fins du droit international privé, pour la qualification extracontractuelle, peut-être discutable du point de vue du droit civil, mais plus respectueuse de l'esprit internationaliste que la qualification contractuelle, nécessitant probablement l'intervention répétée de l'exception d'ordre public. De plus, d'un point de vue fonctionnel, la politique de dissuasion envers les constructeurs négligents bénéficie de la qualification extracontractuelle, même si l'on introduit par là un certain dépeçage dans le contrat ${ }^{86}$. Enfin, en droit positif, l'article 35 de la Loi sur la qualification professionnelle des entrepreneurs de construction et l'arrêt Procon (Great Britain) Ltd peuvent être invoqués au soutien de cette position.

Avant d'examiner les dispositions relatives à la condition des sociétés étrangères qui viendraient soumissionner au Québec, il reste quelques points à préciser, en ce qui concerne la loi applicable à d'autres aspects de l'opération de construction internationale.

\section{Quelques autres aspects conflictuels du droit international privé de la construction internationale}

Quelle est la loi applicable aux rapports de sous-traitance dans le domaine de la construction? (A) Quelle loi régit l'assurance en matière de contrat de construction? (B)

85. Dans le même sens, au sujet de l'article 8.1 C.c., voir H.P. GLENN, «La guerre de l'amiante», (1991) 80 Rev. crit. dr. internat. privé 41, p. 51-54.

86. Sur notion de dépeçage, voir notamment: GROFFIER, Précis, op. cit., supra, note 2, no 33-35, p. 38-39. 


\section{A. La loi applicable à la sous-traitance}

Du point de vue du droit international privé québécois, en ce qui concerne le rapport entre les parties, la question ne présente aucune particularité: la loi d'autonomie s'applique au fond du contrat et à la responsabilité qui en découle. En droit québécois, la responsabilité légale du constructeur ne s'applique pas au sous-traitant qui ne traite pas directement avec le maître d'ouvrage $^{87}$. En droit international privé québécois, à notre avis, cette responsabilité ne devrait donc pas être possible si la construction est érigée au Québec, dans l'hypothèse d'une qualification extracontractuelle de la question. Mais elle pourrait y être reconnue en vertu de la loi étrangère du lieu de construction, si celui-ci est à l'étranger.

Le tribunal internationalement compétent ne sera pas nécessairement le même pour le contrat principal et le sous-contrat puisque l'article 68 C.p.c. comprend plusieurs chefs de compétence alternatifs.

Quant à la loi applicable aux rapports découlant de l'opération de sous-traitance, il n'existe a priori aucune raison pour que le seul fait du choix ou de l'agrément du sous-traitant par le maître d'ouvrage suffise à donner compétence à la loi régissant le contrat principal. Évidemment cette solution est souhaitable pour le maître d'ouvrage et l'entrepreneur général, notamment si les sous-contrats sont «transparents», c'est-à-dire si les obligations du sous-contractant sont calquées sur celle de l'entrepreneur général, pour leur interprétation quant à l'exécution correcte des obligations et pour les causes d'exonération de responsabilité contractuelle ${ }^{88}$.

87. Voir: DURNFORD, précité, supra, note 73, p. 185-186.

88. Sur ces questions, voir notamment: M. E. SCHNEIDER, «International Construction Contracts», (1983) 9 D.P.C.I. 277, 429, 649, p. 301-308; A. BRAIBANT, Le contrat international de construction, Bruylant, Bruxelles, 1981, p. 211 et suiv.; P. LAGARDE, «La sous-traitance en droit international privé» dans C. GAVALDA (dir.) La soustraitance de marchés de travalx et de services, Paris, Économica, 1978, p. 186 et suiv.; dans le même ouvrage, voir M. DAHAN, «Remarques pratiques sur la sous-traitance dans les relations internationales", p. 205 et suiv.

Pour un exemple jurisprudentiel, voir la sentence de la Chambre de commerce internationale, aff. no 2119/78, publiée au Clunet 1979.997 (citée par REMERY, loc. cit., supra, note 3, p. 260). 
On peut cependant remarquer que l'intervention éventuelle de la clause échappatoire, prévue dans le futur Code civil québécois, pourrait mener à effectuer cette liaison en cas de contrats transparents, à défaut de désignation expresse de loi applicable ${ }^{89}$. On pourrait alors rencontrer le problème de l'étendue de la liaison - tout le contrat ou toutes les questions communes? c'est-à-dire celui du dépeçage ${ }^{90}$.

En droit actuel, on appliquerait probablement soit la loi du lieu d'exécution de la construction (si la volonté implicite des parties est suffisamment caractérisée en ce sens) ou, à défaut de toute indication, celle du lieu de conclusion du contrat de sous-traitance (art. 8 C.c.).

La question délicate, en ce domaine, concerne la loi applicable aux rapports entre sous-traitants et maître d'ouvrage ${ }^{91}$ et non celle relative aux rapports entre celui-ci et l'entrepreneur général. En droit québécois à l'inverse du droit français ${ }^{92}$, il n'existe pas d'action directe du sous-traitant ${ }^{93}$, ni d'obligation d'obtenir du maître d'ouvrage l'acceptation du sous-traitant: les conflits de lois peuvent donc se produire en raison de ces différences.

Un juge québécois, dans l'état actuel des choses, ne serait certainement pas prêt à appliquer ces dispositions en tant que lois de police étrangères, si elles étaient considérées de cette nature ${ }^{94}$, et si elles n'étaient pas parties de la loi contractuelle applicable. Le problème est donc de déterminer quelle loi contractuelle doit régir ces questions.

Quant aux questions d'action directe contre le maître d'ouvrage, par exemple pour obtenir le paiement, il nous semble qu'elles devraient être

89. Voir en ce sens LAGARDE, id, p. 190. Sur la notion de clause échappatoire, voir supra, note 8.

90. Id, p. 191.

91. Voir Y. FLOUR, L'effet des contrats à l'égard des tiers en droit international privé, thèse, Paris II, 1977, polycopiée.

92. Loi no 75-1334 du 31 déc. 1975 relative à la sous-traitance, J.O. 3 janvier 1976, 148, D.1976.Lég.64.

93. ROUSSEAU-HOULE, op. cit., supra, note 52, p. 204.

94. P. LAGARDE, (loc. cit., supra, note 88, p. 192 et suiv.) estime qu'il ne s'agit pas de lois de police. 
rattachées à la loi régissant le contrat entre entrepreneur général et sous-traitant car elles concernent directement l'obligation de paiement de l'entrepreneur général $^{95}$. Par contre les recours récursoires du maître d'ouvrage contre l'entrepreneur devraient être régis par la loi applicable au contrat principal.

Il est certain que cette dichotomie peut aboutir à des situations injustes, notamment pour le maître d'ouvrage, d'où l'intérêt, pour lui, d'une liaison entre les lois contractuelles. Mais il est probable que l'exception d'ordre public interviendra si la loi refusant le recours récursoire est une loi étrangère.

Quant à l'obligation pour l'entrepreneur général d'obtenir du maître d'ouvrage l'acceptation du sous-traitant, elle devrait être régie par la loi applicable au contrat principal. Mais ses conséquences éventuelles sur le contrat de sous-traitance devraient être régies par la loi applicable au sous-contrat. Ainsi, si le défaut d'acceptation entraîne la nullité du sous-contrat ou son inopposabilité au maître d'ouvrage, en vertu d'une loi potentiellement applicable, il nous semble que cette question devrait logiquement être soumise à la loi du sous-contrat ${ }^{96}$.

Enfin l'existence du privilège du sous-traitant sur la construction devrait être soumise à la loi contractuelle du sous-contrat ${ }^{97}$, tandis que son opposabilité aux tiers devrait dépendre de la loi du lieu de situation de la construction (art. 6 al.1 C.c.) ${ }^{98}$.

95. Voir dans le même sens, pour l'action directe mais non pour le paiement direct, LAGARDE, id, p. 198; FLOUR, op. cit., supra, note 91, no 83: «la loi de la créance garantie offre ainsi logiquement, non pour la seule action de la victime, mais pour toute action directe, le meilleur rattachement».

96. Contra LAGARDE, id, p. 195.

97. Voir en ce sens LAGARDE, id, p. 200. Pour le privilège du vendeur impayé, voir GROFFIER, Précis, op. cit., supra, note 2, no 195, p. 194, relevant que la jurisprudence a qualifié le privilège de question contractuelle: le raisonnement peut aisément être transposé en notre matière. Comparez avec l'opinion de J.A. TALPIS, «The Law Governing the "Statut Réel" in Contracts for the Transfer Inter Vivos of Moveables "ut Singuli" in Quebec Private International Law», (1972) 13 C. de D. 305, p. 359 (en faveur d'un cumul).

98. Voir LAGARDE, ibid. 
On peut également demander quelle loi régit le contrat d'assurance entre le maître de l'ouvrage ou l'entrepreneur (c'est celui-ci qui a l'obligation de s'assurer selon la pratique québécoise), et l'assureur.

\section{B. La loi applicable au contrat d'assurance}

Ici encore, la loi d'autonomie s'applique en principe. Toutefois le droit québécois présente l'originalité de faire de ses dispositions sur les assurances $^{99}$ d'authentiques lois de police en vertu des articles 2496 et 2500 C.c. ${ }^{100}$ L'originalité est même double, puisque l'article 2496 précise expressément, bien que dans une formule unilatéraliste d'apparence conflictuelle, le domaine d'application international de ces lois de police en dehors desquelles la loi d'autonomie retrouve son domaine ${ }^{101}$. Parmi ces lois de police on trouve certaines règles limitant les clauses exclusives de garantie.

Ainsi, selon l'article 2481 C.c., les clauses générales libérant l'assureur de garantie en cas de violation des lois ou des règlements sont nulles (à moins que la violation ne constitue un acte criminel). Les exclusions de garanties doivent être expresses (art. 2482, 2579 et 2601 C.c.): il en est ainsi notamment pour les exclusions fondées sur le cas fortuit ou la faute de l'assuré (art. 2563 C.c.).

Parmi les nombreuses autres dispositions d'ordre public, on peut citer: l'article 2593 C.c. excluant la responsabilité de l'assureur pour les dommages

99. Voir les articles du Code civil, énumérés à l'article 2500 C.c. (voir note suivante).

100. «Art. 2496. Est réputé conclu au Québec tout contrat d'assurance souscrit par une personne y ayant son domicile ou sa résidence ou portant sur une chose ou un intérêt situé au Québec, dès lors que le preneur en fait la demande au Québec ou que l'assureur y signe ou y délivre la police.

Un tel contrat est régi par la loi du Québec.»

Art. 2500. «Est sans effet toute stipulation qui déroge aux prescriptions des articles 2474 , 2478 à $2484,2486,2490$ à 2492,2494 à 2506 [etc]. [...]

Sauf dans la mesure où elle est plus favorable au preneur ou au bénéficiaire, est sans effet toute stipulation qui déroge aux prescriptions des articles 2485 , [etc.]》

101. Voir Essiambre c. Mougeot, [1976] C.A. 650. Voir à ce sujet CASTEL, op. cit., supra, note 2, p. 535 et suiv.; GROFFIER, Précis, op. cit., supra, note 2, no 209, p. 203; TALPIS, loc. cit., supra, note 28 , p. 215. 
résultant d'incendie ou d'explosion causés par un cataclysme; l'article 2603 C.c. donnant au tiers lésé un droit d'action direct contre l'assureur. En matière de formation du contrat d'assurance, l'article 2478 C.c. oblige l'assureur à remettre un écrit au preneur, indiquant obligatoirement le nom des parties, l'objet et le montant de la garantie, la nature du risque, etc. (art. 2480 C.c.). De même, les clauses libérant l'assureur en cas d'omission ou de fausses déclarations sont réglementées assez strictement (art. 2485 à 2490 C.c.), ainsi que le régime de l'aggravation du risque (art. 2566 C.c.), les conditions de résiliation du contrat (art. 2567 à 2569 C.c.), celles du paiement de la prime (art. 2570 C.c.), de la déclaration de sinistre (art. 2572 à 2574 C.c.) et de paiement de l'indemnité (art. 2575 C.c.). Enfin, d'autres dispositions impératives s'appliquent à chaque type particulier d'assurance de dommages (assurance générale de choses, assurance contre l'incendie et assurance de responsabilité: art. 2579 à 2605 C.c.).

Il faut ajouter qu'en raison de la formulation des articles 2496 et 2500 C.c., toutes les dispositions québécoises sur les assurances seraient théoriquement des lois de police, même s'il s'agit d'articles non impératifs en droit civil interne... En effet, puisque dans les circonstances envisagées par l'article 2496 C.c., la loi québécoise - toute cette loi et non seulement ses dispositions impératives - s'applique sans possibilité de stipulation contraire désignant une loi étrangère, il ne semble pas permis non plus d'écarter ses dispositions facultatives dans ces situations internationales. Mais ces curieuses lois de police permettent quand même aux parties d'y déroger, dans la mesure où les dérogations respectent elles-mêmes le droit québécois. Incidemment, on peut se permettre de douter de la pertinence d'une telle structure: où est l'intérêt vital du Québec dans ces circonstances?

Cependant, dans la pratique québécoise, les assurances ne sont qu'un des moyens d'éviter les risques afférant au contrat d'entreprise, l'autre étant l'usage très répandu des cautionnements ${ }^{102}$. On pourrait donc se demander quelle loi régit cet aspect de l'opération de construction. En ce domaine, en

102. Voir C. DUBREUIL, Rapport canadien au Congrès Capitant, (Journées égyptiennes, 1216 mai 1991), La responsabilité des constructeurs. L'assurance en matière de construction, p. 12 et suiv. 
définitive, la loi d'autonomie s'applique sans intervention de loi impérative spéciale. Toutefois les articles 1938 et 1943 C.c. comprennent des règles matérielles à but international, obligeant celui qui doit fournir caution à en présenter une «qui ait dans le Bas Canada des biens suffisants pour répondre de l'objet de l'obligation et dont le domicile soit dans les limites du Canada». Elles exigent de la caution invoquant le bénéfice de discussion, de n'indiquer au créancier que des biens appartenant au débiteur principal, situés au Québec. À notre avis, ces dispositions ne s'appliquent que si la loi québécoise régit le contrat de cautionnement ${ }^{103}$.

En terminant l'étude de ces questions particulières, on doit rappeler incidemment qu'un tribunal québécois appliquera impérativement la loi québécoise - intervenant comme loi de police - aux questions de responsabilité résultant de l'exposition à des matières premières produites au Québec, ou de leur utilisation, en vertu de l'article 8.1 C.c. ${ }^{104}$

\section{La condition des sociétés de construction étrangères au Québec}

En droit québécois, les contrats de construction privée ${ }^{105}$ sont régis par les dispositions du Code civil relatives au louage d'ouvrage (art. 1683 à 1697d) C.c.), par des dispositions générales applicables aux obligations, et par les règles du contrat lui même, normalement très détaillées et inspirées de contrats types proposés par les associations. Toute la procédure de soumission et d'adjudication ${ }^{106}$ est réglementée par une entente entre corporations professionnelles, ayant créé en 1967 un «Code provincial du Bureau des

103. Voir en ce sens, en général, CASTEL, op. cit., supra, note 2, p. 24: «Ces règles ne sont pas attributives de compétence». Sur la notion de règles matérielles à but international, voir: GROFFIER, Précis, op. cit., supra, note 2, no 27, p. 31; J.A. TALPIS, «Material Rules of Private International Law in Force in the Province of Quebec», (1973) 8 R.J.T. 223.

104. Voir à ce sujet H.P. GLENN, loc. cit., supra, note 85.

105. Sur ce sujet, voir l'étude de ROUSSEAU-HOULE, op. cit., supra, note 52 . En ce qui conceme les contrats publics, pour toutes les règles de droit public qui les régissent en général, nous renvoyons le lecteur à cet ouvrage très exhaustif (417 p. et bibliographie).

106. Sur l'adjudication, en plus de l'ouvrage de ROUSSEAU-HOULE; id., p: 78 et suiv.), voir l'ouvrage récent de C. MOFFET, L'entrepreneur général, les sous-traitants et le Bureau des soumissions déposées du Québec, Montréal, Wilson \& Lafleur, 1990. 
soumissions déposées du Québec» ${ }^{107}$. Il s'agit d'une entente contractuelle pour les donneurs d'ordre, qui ne sont pas obligés de s'y référer, mais de nature obligatoire, pour les membres des corporations professionnelles d'entrepreneurs $^{108}$, quant aux travaux concernés. Pour ces derniers «toute violation de l'entente devient une violation de la lois ${ }^{109}$, sanctionnée par des peines disciplinaires et des amendes civiles $^{110}$.

La détermination du domaine d'application des règles du Bureau des soumissions déposées du Québec (ci-après B.S.D.Q.) est assez complexe ${ }^{111}$. On peut d'abord s'y référer pour des raisons d'opportunité (mais alors ses règles deviennent obligatoires pour les parties) ${ }^{112}$. Elles s'appliquent aussi, d'office cette fois, à tous les travaux visés qui doivent être exécutés au Québec ${ }^{113}$ par les personnes assujetties à ses règles.

Les travaux visés sont ceux pour lesquels plus d'une offre est demandée et dont le prix de soumission dépasse $10000 \$ \$ 114$. Évidemment, leur nature (électricité, plomberie; etc.) détermine aussi leur assujettissement $^{115}$.

Les personnes assujetties aux règles du B.S.D.Q. sont les suivantes: le donneur d'ordre peut y recourir volontairement; les membres des corporations professionnelles qui font partie de l'entente; les membres d'autres associations $^{116}$ auxquelles l'entente a été étendue; et les non-membres, qui

107. MOFFET, id., p. 2 et suiv.

108. MOFFET, $i d$, p. 19 et suiv.

109. MOFFET, id, p. 4, citant Robertson C. C.M.E.Q., D.T.E. 87 T-404, (C.A.) p. 4 du jugement.

110. MOFFET, id, p. 4 et p. 67 et suiv.

111. Voir art. B-2 du Code du B.S.D.Q.; MOFFET, id, p. 8-26; ROUSSEAU-HOULE, op. cit., supra, note 52 , p. 78 et suiv.

112. MOFFET, id, p. 9 et p. 22-25; ROUSSEAU-HOULE, id, p. 79-80.

113. Art. B-2 paragraphe 1 du Code de B.S.D.Q.; voir MOFFET, id., p. 25-26.

114. Art. B-2 paragraphe 3 du Code de B.S.D.Q.

115. MOFFET, op. cit., supra, note 106, p. 13-16; ROUSSEAU-HOULE, op. cit., supra, note 52, p. 78 .

116. MOFFET, id., p. 19-22. Mis à part le cas des travaux assujettis au Code par résolutions spéciales (id., p. 21). 
éventuellement effectueraient de tels travaux ${ }^{117}$, bien qu'ils n'en aient théoriquement pas le droit sans être affiliés à ces corporations ${ }^{118}$.

Quant aux sociétés étrangères de construction, on doit noter qu'il existe de nombreux obstacles à leur admission à la procédure d'adjudication au Québec, si bien qu'en pratique elles n'y sont pas présentes en tant que telles et les soumissionnaires choisis sont quasiment toujours des sociétés québécoises.

La procédure permettant toutefois de participer à l'adjudication consiste soit à créer une filiale québécoise, enregistrée comme toute autre compagnie locale, soit à obtenir une licence comme société étrangère, puis à créer une joint venture avec une société locale québécoise.

Il faudra donc, dans un premier temps, se conformer aux dispositions de la Loi sur les compagnies étrangères ${ }^{119}$. En vertu de cette loi, aucune compagnie étrangère ne peut faire d'affaires au Québec sans obtenir d'abord un permis ${ }^{120}$ qui sera accordé si la société 1 ) dépose une copie certifiée de sa charte au Québec, ainsi qu'une procuration y constituant un agent principal pour y recevoir toute signification, 2) paye certains honoraires, 3) établit qu'elle est constituée de manière à remplir les obligations qu'elle peut contracter et 4) se conforme à la politique linguistique québécoise quant à sa dénomination sociale ${ }^{121}$. La corporation étrangère pourra alors faire affaires au Québec dès la publication au joumal officiel de son avis d'autorisation ${ }^{122}$, mais l'étendue de ses pouvoirs dépend du permis, des limites de sa propre charte et éventuellement d'autres restrictions légales particulières ${ }^{123}$.

117. MOFFET, id, p. 13, note 42 .

118. MOFFET, id. p. 13.

119. L.R.Q. c. C-46.

120. Art. 3, L.R.Q., c. C-46.

121. Art. 4, L.R.Q., c. C-46.

122. Art. 5, LR.Q., c. C-46.

123. Art. 8, L.R.Q., c. C-46. Voir sur les pouvoirs des sociétés étrangères au Québec, en général: J.A. TAIPIS, «Aspects juridiques de l'activité des sociétés et corporations étrangères au Québec», [1976] C.P. du N. 215; J. TASCHEREAU, «Conflits de lois en matière de sociétés civiles et commerciales» dans Mélanges offerts à Me Raymond Cossette, Québec, Chambre des notaires, 1968 , p. 143 et CASTEL, op. cit., supra, note 
En ce qui concerne l'entreprise de construction étrangère, ces dispositions spéciales proviennent d'abord de l'article 35 de la Loi sur la qualification professionnelle des entrepreneurs de construction ${ }^{124}$. Il dispose que la société dont le principal établissement est situé hors du Québec, qui désire y exécuter ou $\mathrm{y}$ faire exécuter par sous-traitance des travaux de construction, doit obtenir une licence temporaire spéciale et fournir un cautionnement pour fraude, faillite ou insolvabilité (art. 34 et 34.1), comme tout autre entrepreneur du Québec.

Sans cette licence, l'entreprise étrangère ne peut exercer d'activité de construction au Québec (art. 25 de la loi). Elle s'obtient si au moins une personne physique, participant à la gestion de la société, a obtenu elle-même une licence auprès de la Régie des entreprises de construction du Québec, à la suite d'examens démontrant que la personne 1) possède les connaissances ou l'expérience requises dans la gestion d'une entreprise de construction et l'exécution de travaux de ce genre, 2) établit sa solvabilité, 3) prouve qu'elle n'a pas été condamnée depuis 5 ans et qu'elle est inscrite régulièrement à la corporation professionnelle pertinente (art. 31 de la Loi) ${ }^{125}$. Ce sont donc normalement des Québécois qui habilitent la société étrangère ou la joint venture décidée à construire au Québec.

De plus, la société étrangère demandant la licence temporaire doit élire un domicile au Québec (art. 35 de la loi), ce qui peut se faire simplement par le biais d'une domiciliation chez un avocat ou un notaire.

2, p. 333 et suiv. spécialement p. 340-343; GROFFIER, Précis, op. cit., supra, note 2, no 149 , p. 147. Voir aussi, A. PRUJINER, «La personnalité morale et son rattachement en droit international privé», (1990) 31 C. de D. 1049, 1067; J.A. TALPIS, «La représentation volontaire et organique en droit international privé québécois», (1989) 20 R.D.U.S. 89.

124. Précitée, supra, note 46.

125. Voir le Règlement de la Corporation des mâ̂tres électriciens du Québec, R.R.Q. 1981, c. $M-3, I-1$, art. 11 prévoyant les conditions suivant lesquelles un entrepreneur étranger peut obtenir un certificat de membre temporaire de la Corporation (examen, paiement d'honoraires, respect des règlements de la Corporation). Pour les maîtres mécaniciens en tuyauterie, voir le Règlement de la Corporation des maîtres mécaniciens en tuyauterie du Québec, R.R.Q. 1981, c. M-4, r-1, art. 63, prévoyant les mêmes conditions. 
Comme tout employeur au Québec, elle doit aussi respecter la législation du travail et de la sécurité sociale ${ }^{126}$, c'est-à-dire payer des cotisations à la Commission de la sécurité et de la santé du travail pour ses employés, faire partie de l'association des entrepreneurs en construction du Québec, détenir des certificats de compétence professionnelle ${ }^{127}$ et se conformer à toutes les autres lois locales pertinentes de droit public.

Naturellement, les ingénieurs et les architectes habilités à exercer leur profession au Québec doivent appartenir aux corporations professionnelles québécoises ${ }^{128}$. L'Ordre des ingénieurs peut cependant accorder un permis temporaire, à titre de collaborateur ou même comme ingénieur, à une personne non domiciliée au Québec $^{129}$. On retrouve la même possibilité pour les architectes $^{130}$.

De toutes façons, comme nous l'avons dit plus haut, il est très rare en pratique, que des sociétés étrangères opèrent dans le domaine de la construction privée au Québec.

Pour les contrats de construction donnés par le gouvernement et les organismes publics québécois ${ }^{131}$, la perspective est encore plus sombre.

En effet, une décision du Conseil des ministres de $1977^{132}$ énonce une politique selon laquelle les ministères et les organismes publics devront effectuer les achats sur une base sectorielle et régionale lorsque cette politique

126. Loi sur la santé et la sécurité du travail, L.R.Q., c. S-2.1. (refonte actuelle)

127. Voir la Loi sur les relations du travail, la formation professionnelle et la gestion de la main-d'oeuvre dans l'industrie de la construction, L.R.Q., c. R-20; Loi sur la formation et la qualification professionnelle de la main-d'oeuvre, L.R.Q., c. F-5.

128. Loi sur les ingénieurs, L.R.Q., c. I-9, art. 6; Loi sur les architectes, L.R.Q., c. A-21, art. 2.

129. Id., art. 19 et 20.

130. Voir Code des professions, L.R.Q., c. C-26, art. 32, 33, 39 et 41 (permis temporaire).

131. Voir P. GIROUX, C. MOFFET, D. LEMIEUX et M.-C. BELLEAU, Contrats des organismes publics québécois, vol. 1, Farnham, CCH/FM Ltée, 1988 (et mises à jour).

132. Décision du Conseil des ministres, no 77-19 du 26 janvier 1977, citée dans GIROUX, MOFFET, LEMIEUX et BELLEAU, id, p. 935. 
bénéficiera à l'économie québécoise ${ }^{133}$. En conséquence, des mesures protectionnistes ont été adoptées. La politique d'achat prévoit que s'il existe, dans un secteur économique donné, au moins trois entreprises québécoises, seules les entreprises québécoises peuvent soumissionner ${ }^{134}$.

De plus, des dispositions obligent les organismes publics à formuler leurs appels d'offre favorisant «les solutions qui représentent le plus fort contenu québécois» et donnant "priorité à l'utilisation de produits québécois» ${ }^{135}$. On y précise que s'«il existe des produits fabriqués au Québec, seuls ces produits et matériaux doivent être spécifiés au cahier des charges» ${ }^{136}$. Lorsqu'il n'en existe pas, «la préférence doit être accordée aux produits canadiens et, si possible, achetés au Québec» ${ }^{137}$. On y prévoit des clauses devant obligatoirement apparaître dans les appels d'offre, spécifiant que seules seront prises en considération:

«les soumissions des entrepreneurs ayant leur principale place d'affaires au Québec (c'est-à-dire, le principal établissement d'où les affaires sont dirigées et où le personnel de maîtrise et l'équipement se trouvent ordinairement) et détenant la licence requise en vertu de la Loi sur la qualification professionnelle des entrepreneurs de construction.» ${ }^{138}$

Ces conditions s'appliquent aussi aux sous-traitants retenus par le soumissionnaire choisi ${ }^{139}$. Leur violation peut entraîner des pénalités ${ }^{\mathbf{1 4 0}}$.

133. GIROUX, MOFFET, LEMIEUX et BELLEAU, ibid.

134. GIROUX, MOFFET, LEMIEUX et BELLEAU, id, p. 935.

135. Modalité d'application de la politique d'achat dans la construction, C.T. 149240 du 13 mars 1984, art. 1a) et b), reproduits dans GIROUX, MOFFET, LEMIEUX et BELLEAU, id, p. 10,651 et suiv.

136. II., art. 2.1.1. [Modalité]

137. Id, art. 2.1.2. [Modalité]

138. Id, art. 2.2.1. [Modalité]

139. GIROUX, MOFFET, LEMIEUX et BELLEAU, op. cit., supra, note 131, p. 934, Règlement sur les contrats de construction du gouvernement, R.R.Q. 1981, c. A-6, r-7, art. 12h) et 14i). 
Enfin, dans les cas d'appel d'offre sur invitation, un fichier d'entrepreneur est constitué et il est prévu que n'y sont inscrits que les entrepreneurs qui:

«a) ont une place d'affaires au Québec comportant des installations permanentes et le personnel de maitrise requis pour diriger les travaux qui font l'objet d'un mandat; et

b) détiennent, s'il y a lieu, une licence émise par la Régie des entreprises de construction du Québec.» ${ }^{141}$

\section{Conclusion}

Comme on peut le constater, le marché québécois de la construction est fortement protégé.

Si les entreprises québécoises se sont déjà engagées dans le marché international de la construction, la prise en compte globale du phénomène par le droit international privé québécois s'impose en raison de son importance économique croissante et nous espérons que cette étude aura contribué à cette prise de conscience.

De ce point de vue, le problème central reste celui de la qualification, contractuelle ou extracontractuelle, de la responsabilité du constructeur pour vice de construction et du sol, car le régime applicable en dépend directement. Si la qualification «naturelle» de cette question passe nécessairement par la fin

140. GIROUX, MOFFET, LEMIEUX et BELLEAU, ibid; Voir le document cité note 135, art. 2.2.2, disposant: «[1]e soumissionnaire ne doit engager que des sous-traitants ayant un établissement comportant au Québec des installations permanentes et le personnel requis [...], à moins [qu'il] fasse la preuve à la satisfaction du propriétaire qu'il n'existe pas au Québec de sous-traitants dans cette spécialité; dans le cas où l'entrepreneur ne peut faire la preuve requise à la satisfaction du propriétaire, ce dernier peut exiger que l'entrepreneur choisisse un sous-traitant du Québec sans changer le prix global de sa soumission".

141. Directive concernant certaines modalités d'application du Règlement sur les contrats de construction du gouvernement, no 1-79 (refondue par le C.T. 142590 du 25 janvier 1983, modifiée par C.T. 149043 du 28 février 1984, C.T. 149360 du 13 mars 1984, C.T. 167326 du 26 avril 1988), art. 6a) et b), reproduite dans GIROUX, MOFFET, LEMIEUX et BELLEAU, id., p. 10,201 et suiv. 
du débat en droit interne, une qualification fonctionnelle, téléologique, nous semble clairement favoriser la thèse extracontractuelle, aux fins du droit international privé. Une règle de conflit spécifique à la matière pourrait être rédigée de la façon suivante:

La responsabilité civile du constructeur pour vice de construction est régie par la loi de l'État dans lequel le constructeur a son établissement principal, à défaut, sa résidence, ou par la loi du lieu de la construction, selon celle qui est la plus favorable au mâtre d'ouvrage.

Dans cette optique extracontractuelle, le choix de la loi reposerait sur le juge.

Dans l'hypothèse d'une qualification contractuelle, il suffirait de laisser ce choix au maître d'ouvrage ou à l'acheteur postérieur de la construction, pour respecter le principe de la loi d'autonomie tout en le limitant. Alternativement, toujours dans cette même hypothèse mais dans une optique plus conciliatrice entre le principe de la loi d'autonomie et le respect de la politique de protection du maître d'ouvrage, on pourrait s'inspirer des formules des articles 3117 et 3118 du Projet de loi $125^{142}$ pour adopter les règles suivantes:

Le choix par les parties de la loi interne applicable au contrat de construction ne peut avoir pour résultat de priver le maître d'ouvrage de la protection que lui accordent les dispositions impératives, relatives à la responsabilité civile du constructeur pour vice de construction, de la loi du lieu de la construction.

En l'absence de désignation par les parties, la loi du lieu de la construction s'applique à la responsabilité civile du constructeur pour vice de construction.

142. Précité, supra, note 6. 